\title{
Delivery of MMP-12 siRNA with a Nanocarrier Improves the Homeostasis of the Small Intestine and Metabolic Dysfunction in High-Fat Diet Feeding- Induced Obese Mice
}

\section{Mingming Song}

China Pharmaceutical University

Shiyao Zhang

China Pharmaceutical University

\section{Zixuan Tao}

China Pharmaceutical University

\section{Yujie Shi}

China Pharmaceutical University

\section{Yonghong Xiong}

China Pharmaceutical University

\section{Wenxiang Zhang}

China Pharmaceutical University

\section{Jianning Li}

Nanjing Qixia Hospital

\section{Chang Liu}

China Pharmaceutical University https://orcid.org/0000-0001-9251-708X

Siyu Chen ( $\square$ siyuchen@cpu.edu.cn )

China Pharmaceutical University

\section{Article}

Keywords: Small Intestine Regulator, Overnutrients, Nano Delivery System, Lipid Transportation, Bile Acid Reabsorption, Inflammation, Epigenetic Modification

Posted Date: November 13th, 2020

DOI: https://doi.org/10.21203/rs.3.rs-99824/v1

License: (9) This work is licensed under a Creative Commons Attribution 4.0 International License. Read Full License 


\section{Abstract}

The changes of small intestinal homeostasis have been recognized to contribute essentially to the obese development. However, the core small intestinal regulator which mediates overnutrient impacts on the homeostasis of the small intestines remains elusive. Here, we identify the MMP-12 as such a responsive factor in mouse small intestines. Taking advantages of the nano delivery system, we demonstrate that small intestine-specific MMP-12 knockdown alleviates high-fat diet feeding-induced metabolic disorders and improves intestinal homeostasis in mice, including a significant decrease in lipid transportation, bile acid reabsorption, and inflammation. In parallel, the small intestinal integrity is recovered and the gut microbiota composition is reversed towards that under normal diet feeding. Mechanistically, MMP-12, differing from its traditional elastolytic function, acts as a transcriptional factor to activate Fabp4 transcription through epigenetic modification. In translational medicine, clinical applications of our nanosystem and therapeutic interventions targeting MMP-12 will benefit patients with obesity and associated diseases.

\section{Introduction}

With the development of the global social economy in the $21^{\text {st }}$ century, obesity has become a global pandemic disease ${ }^{1,2}$. Over the last 40 years, the prevalence of obesity has almost tripled worldwide ${ }^{3}$. The development of obesity is closely associated with multiple diseases, such as type 2 diabetes, hepatic steatosis, cardiovascular diseases, and even cancer ${ }^{4-9}$. Hence, obesity is a great threat to public health and causes an economic burden for society. Although effective strategies, including lifestyle intervention, conventional pharmacology and weight-loss surgery, are well established to counteract obesity, their undesirable inconstancy and side effects are not exempt in all cases ${ }^{7,10}$. Therefore, an urgent medical need exists for the large and heterogeneous population of obese patients.

Although obesity is caused by various pathological factors, changes in the small intestinal homeostasis have been recognized to contribute essentially to obesity progression ${ }^{11}$. As the first organ exposed to nutrients, the small intestine has to change its capacity to adapt external nutrient signals, such as a HFD $^{12}$. When challenged with dietary fat, our stomach functions as a bioreactor for dietary lipid peroxidation. These lipids are further engulfed by enterocytes within the duodenum and are re-esterified into complex lipid molecules. ${ }^{4}$ Then, they bind with lipoproteins (e.g., ApoB48 and ApoB100) and are assembled into primarily chylomicrons in the jejunum ${ }^{13}$. These chylomicrons enter the lymphatics through lacteals and further drain into the venous circulation ${ }^{14,15}$. Subsequently, the ileum plays a nonredundant role in handling lipid overflow by controlling bile acid (BA) reabsorption, cholesterol absorption and metabolism ${ }^{16-18}$. In addition, disruptions of lipid homeostasis can lead to unexpected intestinal inflammation and permeability destruction, as well as alterations in the composition of the intestinal microbiota ${ }^{19-21}$. Collectively, the coordination of such processes in the whole intestine guarantees metabolic homeostasis within our bodies. However, diets with high fat have been associated with various metabolic syndromes, including obesity and diabetes mellitus $4,10,22$. These HFD feeding- 
induced detrimental effects are ascribed to their profound impacts on the overall intestinal homeostasis as stated above. Thus, correction of the small intestinal homeostasis has become a promising approach to treat obesity.

To screen out the direct mediator that transfers HFD signals to intestinal homeostasis, we clustered highthroughput RNA-seq results from the jejunum and ileum (two major organs for lipid absorption) collected from mice subjected to 11 weeks of HFD feeding. We found that the mRNA expression levels of MMP-12 were markedly elevated in both small intestinal tissues. MMP-12 was first demonstrated as an inflammatory macrophage-secreted elastolytic MMP ${ }^{23}$. In addition, MMP-12 is able to degrade a broad spectrum of extracellular matrix (ECM) components, such as collagen IV, fibronectin and laminin. Hence, inflammation-induced MMP-12 degrades the basement membrane, allowing macrophages to penetrate into injured tissue ${ }^{24}$. Accordingly, the expression and enzymatic activity of MMP-12 are increased in multiple inflammatory diseases, including atherosclerosis and chronic obstructive pulmonary disease $\mathrm{e}^{25}$. Importantly, MMP-12 is involved in obesity development. For example, MMP-12 is highly expressed in the adipose tissue of HFD-fed mice. MMP-12 deficiency alleviates obesity-induced inflammation and improves metabolic dysfunction. Such a beneficial effect may be dependent on the organization and composition of the ECM, raising the possibility that MMP-12-orchestrated ECM deposition and degradation are involved in maintaining metabolic homeostasis. Notably, intestinal ECM homeostasis is abolished in HFD-fed mice. Hence, identification of the role of MMP-12 in the regulation of small intestinal function is of great urgency in the treatment of obesity.

In the present study, to effectively and specifically knock down MMP-12 in mouse small intestines, we established a new nanoparticle (NP) system consisting of chitosan (CS), PLGA (poly(lactic-coglycolic acid)) and polyethylene glycol (PEG) (CS@PLGA NPs, CPA NPs) to specifically deliver MMP-12 siRNAs into mouse small intestines. By making the best of this NP system, we found that it improved the small intestinal homeostasis of HFD feeding-induced obese mice. As a consequence, these mice exhibited improved metabolic physiology and reduced systemic inflammation. Mechanistically, MMP-12 exerted DNA-binding abilities, rather than its elastolytic activity, to induce fatty acid-binding protein 4 (Fabp4) transcription. Taken together, we demonstrated a mediatory role of MMP-12 in transferring HFD signals to small intestinal homeostasis and systemic metabolic homeostasis.

\section{Results}

MMP-12 responses to HFD signals in mouse small intestines. To address the aforementioned question, we performed high-throughput RNA sequencing by using small intestinal samples (including jejunum and ileum) dissected from 11-week HFD-fed mice. Bioinformatic analysis revealed a cluster of 8 genes that were robustly regulated by HFD signals in both the mouse jejunum and the ileum (Fig. 1a, b and Supplementary Table 1), which was confirmed by independent RT-qPCR and semiquantitative analyses (Fig. 1c, Supplementary Fig. 1a and Supplementary Table 2). Moreover, in vitro analysis revealed that 0.4 $\mathrm{mM}$ free fatty acids (FFAs, an equimolar mixture of oleic acid and palmitic acid) increased the mRNA expression levels of MMP-12, LEP and CYP1A1 in Caco-2 cells, a colorectal adenocarcinoma-derived cell 
line. However, other genes, including GPR17 and KCNJ14, were modestly altered by such stimulation (Fig. 1d). Given that MMP-12 was the most responsive gene corresponding to the hyperlipidemic signals in vivo and in vitro, we ultimately focused our research on this gene. Consistently, the protein expression levels of MMP-12 were similarly increased (Fig. 1e and Supplementary Fig. 1b, c).

Of note, MMP-12 is also a macrophage metal elastase that possesses biological and pathophysiological functions based on its enzymatic activity. Hence, we evaluated the enzymatic activities of MMP-12 and found that they were consistently increased in the jejunum and ileum of HFD-fed mice, as well as in FFAtreated Caco-2 cells (Fig. $1 \mathrm{f}$ and Supplementary Fig. 1d, e). Moreover, MMP-12 is known as a secretory protease that degrades the ECM during the destruction of inflammatory tissues. As shown in Fig. 1g, the serum and fecal levels of MMP-12 were correspondingly increased in HFD-fed mice. Meanwhile, FFAs also increased the MMP-12 concentrations in the supernatant from Caco-2 cells (Fig. 1h).

Taking these data together, we concluded that MMP-12 may serve as an important factor responding to HFD signals in the small intestine.

Preparation and Characterization of CPA NPs. To specifically and effectively knock down MMP-12 in the small intestines of HFD-fed mice, we designed an oral NP system using PLGA-PEG and CS based on the $\mathrm{pH}$-sensitive and adhesive effects of CS. In brief, PLGA-PEG was first synthesized by conjugating $\mathrm{COOH}$ PEG-NH2 to PLGA-N-hydroxysuccinimide (PLGA-NHS) through amide bond formation (Fig. 2a). In the ${ }^{1} \mathrm{H}$ nuclear magnetic resonance (NMR) spectra of PLGA-PEG, the peaks at 5.2, 4.8, 3.7, and $1.6 \mathrm{ppm}$ were ascribed to (3, $-\mathrm{OC} \underline{\mathrm{H}}-),\left(2,-\mathrm{O} \underline{\mathrm{H}}_{2}-\right),\left(4,-\mathrm{CH}_{2}-\mathrm{CH}_{2} \mathrm{O}-\right)$, and $\left(5,-\mathrm{OCH}\left(\mathrm{C}_{3}\right)-\right)$, respectively (Fig. 2b). The degradable CPA NPs were prepared using PLGA-PEG polymer (as a core) coated with a CS shell (as a protective shelter to avoid gastric acid) by double-emulsion and ionic gelation strategies (Fig. 2c). The CPA NPs were roughly spherical in shape and had a particle diameter of $\sim 227 \mathrm{~nm}$, as analyzed by transmission electron microscopy (TEM) and light scattering spectrometry (Fig. 2d). The polymer disparity index (PDI) and zeta potential were $0.23 \pm 0.01$ and $57.8 \pm 4.98 \mathrm{mV}$, respectively (Fig. 2e). Essential to the NP system, the protonation of the CS shell stabilized the PLGA-PEG NPs under acidic conditions (Fig. 2f). Next, cationic poly-L-lysine (PLL) was used for siRNA complexation (PLL/siRNA weight ratio of 0.5 , Fig. $2 \mathrm{~g}$ ). In contrast, this shell collapsed in the simulated intestinal fluid (SIF), an in vitro system that mimics the basic environment in the small intestines (Supplementary Table 3) ${ }^{26}$.

Endocytosis has been well recognized as an important process through which extracellular cargos are internalized by cells ${ }^{27}$. To identify the mechanisms through which the CPA NPs entered the intestinal cells, we treated human Caco-2 cells with CPA NPs carrying FAM siRNA (with green fluorescence) for $4 \mathrm{~h}$ in the presence of different inhibitors for the endocytic process. As shown in Fig. 3a, CPA NP transfection was partially blocked by treatment with either EIPA (a macropinocytic inhibitor) or chlorpromazine (a clathrin-mediated endocytic inhibitor). Notably, cellular uptake was dramatically diminished in combination with filipin (a caveolae-mediated endocytic inhibitor). Taken together, these results 
suggested that caveolae-mediated endocytosis is the predominant pathway for the cellular uptake of CPA NPs. In addition, the CPA NP-coated siRNA was more prone to absorption than the PLGA NPs (Fig. 3b), which could be explained by the stronger positive electricity of the CPA NPs.

To evaluate the biodistribution of CPA NPs, Cy 5 was grafted onto PLGA to form fluorescent NPs for in vivo tracking (Fig. 3c, d). Next, the mice were gavaged with Cy5-labeled CPA NPs ( $40 \mu \mathrm{g} / \mathrm{kg}$ ) and equivalent Cy5-labeled PLGA NPs for $48 \mathrm{~h}$. We found that the fluorescent signals of Cy5-labeled CPA NPs accumulated in mouse small intestines and exhibited retentive ability compared with that of the Cy5labeled PLGA NPs (Fig. 3e-g). In addition, we did not observe any fluorescent signals in other metabolic organs, including the liver, epididymal white adipose tissue (eWAT), kidney, or spleen (Fig. 3h).

Biosafety analysis of CPA NPs. To investigate the potential toxic effects of CPA NPs, we treated Caco-2 cells with different components or doses of CPA NPs. We found that none of them affected the cell viability of Caco-2 cells, indicating that CPA NPs were safe for intestinal cells (Supplementary Fig. 2a, b). More importantly, CPA NPs modestly affected the mouse body weight and food intake when compared to the PBS-treated group (Supplementary Fig. 3c, d). In addition, we did not observe any changes in serological hepatic injury parameters, including alanine aminotransferase (ALT) and aspartate transaminase (AST) (Supplementary Fig. 3e). Similar results were observed for renal injury markers, such as serum creatinine and blood urea nitrogen (BUN) (Supplementary Fig. 2f, g). Histologically, we found that no significant differences existed in any examined organs between groups (Supplementary Fig. $2 \mathrm{~h}$ ).

\section{MMP-12-siRNA-CPA NPs improve HFD feeding-induced obesity and insulin resistance. To further identify} the specific role of MMP-12 in mouse small intestines, we used a state-of-art CPA NP system carrying an MMP-12 siRNA mixture to construct mice with small intestine-specific MMP-12 knockdown (with $93.46 \pm$ 0.54\% encapsulation efficiency, Supplementary Fig. 3a). The CPA NP-mediated knockdown efficiency and specificity are shown in supplementary Fig. 3b-e. Note that enzyme-linked immunosorbent assay (ELISA) analysis revealed that knockdown of MMP-12 in mouse small intestines significantly reduced the serum and fecal levels of MMP-12, indicating that the small intestine was a dominant organ for MMP-12 secretion (Supplementary Fig. 3f). As shown in Fig. 4a, b, HFD feeding-induced body weight gain was attenuated by $19.86 \%$ in mice with oral administration of MMP-12-siRNA-CPA NPs, while food intake was modestly altered by MMP-12-siRNA-CPA NPs in HFD-fed mice (Fig. 4c). Since the small intestine is an important organ for maintaining lipid homeostasis and contributes essentially to obese development, we determined the impact of MMP-12 knockdown on whole-body energy metabolism using metabolic cage analysis. We found that administration of MMP-12-siRNA-CPA NPs significantly increased $\mathrm{O}_{2}$ consumption and $\mathrm{CO}_{2}$ production in HFD-fed mice (Fig. 4d-f). Consistently, HFD feeding-induced hypertriglycemia, hypercholesterolemia, fasting hyperglycemia, hyperinsulinemia, the increased homeostasis model assessment (HOMA) of insulin resistance (HOMA-IR), and hyperlipidemia were 
alleviated in these mice (Fig. $4 \mathrm{~g}$ and Supplementary Fig. 3g, h). In contrast, the fecal levels of total cholesterol (TC) and nonesterified fatty acids (NEFAs) were correspondingly increased (Supplementary Fig. 3i). In parallel, glucose intolerance and insulin sensitivity were correspondingly improved by MMP-12 knockdown (Fig. 4h, i and Supplementary Fig. 3j). These results suggested that the overall metabolic disorders were attenuated by MMP-12 manipulation.

\section{MMP-12-siRNA-CPA NPs ameliorate HFD feeding-induced hepatic steatosis, adiposity and systemic} inflammation. Given that impaired insulin sensitivity facilitates the pathological progression of hepatic steatosis, adiposity and systemic inflammation ${ }^{28}$, we next examined the effects of MMP-12-siRNA-CPA NPs on these pathological parameters in HFD-fed mice. As shown in Fig.5a (Supplementary Fig. 4a, b), MMP-12-siRNA-CPA NP administration alleviated HFD feeding-induced hepatic and epididymal fat accumulation. Histological analysis revealed that large lipid droplets observed in liver and epididymal fat sections isolated from HFD-fed mice were significantly shrunk by MMP-12-siRNA-CPA NPs (Fig. 5b and Supplementary Fig. 4c, d). Notably, the liver contents of triglycerides (TG) and TC, as well as serum levels of transaminases, were decreased in these mice compared to the HFD-fed mice (Fig. 5c, d). At the inflammatory level, MMP-12-siRNA-CPA NP treatment significantly decreased HFD feeding-induced macrophage infiltration in the liver, eWAT and subcutaneous white adipose tissue (sWAT), as evidenced by immunohistochemical (IHC) analysis of $\mathrm{F} 4 / 80$ expression (Fig. 5e). Consistently, serological analysis of inflammatory cytokines, including interferon-6 (IL-6), tumor necrosis factor-a (TNF-a), and monocyte chemotactic protein-1 (MCP-1), were decreased in these mice treated with MMP-12-siRNA-CPA NPs (Fig. 5f). These results confirmed that HFD feeding-induced systemic inflammation was ameliorated by MMP12-siRNA-CPA NP treatment.

\section{MMP-12-siRNA-CPA NPs improve lipid transportation and BA reabsorption in the small intestines of HFD-}

fed mice. To identify the potential mechanism underlying the protective effects of MMP-12 knockdown in the mouse small intestines on diet-induced obesity, we next performed transcriptional profiling on small intestine samples (a mixture of jejunum and ileum) to globally screen out the downstream events of MMP-12. As shown in Fig. 6a, MMP-12 knockdown increased the expression levels of 630 genes. In contrast, the expression levels of 454 genes were decreased. Gene ontology (GO) analysis revealed a cluster of genes involved in lipid homeostasis (e.g., lipid uptake/transportation and BA reabsorption) and the immune response (Fig. 6b). Hence, we examined whether MMP-12 was essential for the transport of dietary lipid absorption into the circulation since the small intestine is an important organ for such a process. As shown in Fig. 6c, postprandial TG response assays indicated that administration of MMP-12siRNA-CPA NPs markedly decreased serum TG levels in HFD-fed mice. Histological and Nile red staining analyses indicated that refeeding-induced lipid accumulation in the enterocytes of the mouse small intestines was almost absent in these mice (Fig. 6d). 
Of note, chylomicron contributes importantly to mediating the transportation of dietary lipids into the circulation system. ${ }^{14}$ Transmission electronic microscopy (TEM) scanning analysis indicated that smaller chylomicron particles existed in the serum from MMP-12 knockdown mice after 2-h HFD refeeding compared to the control group (Fig. 6e and Supplementary Fig. 5a, b). Coincident with these findings, the serum collected from $2 \mathrm{~h}$ HFD-refed mice administered MMP-12-siRNA-CPA NPs was transparent, whereas the control serum was milky (Fig. 6e). At the molecular level, ApoB-48 contents in the chylomicron fraction and serum were correspondingly reduced, while its expression was consistently reduced in mouse small intestines (Fig. 6f, Supplementary Fig. 5c).

BAs are involved in the absorption and metabolism of dietary lipids in the small intestines, as well as gut permeability and bacterial composition. ${ }^{21}$ As shown in Fig. $6 \mathrm{~g}$, we found that serum levels of total BAs were decreased by approximately $45.15 \%$ in HFD-fed mice treated with MMP-12-siRNA-CPA NPs compared to HFD-fed mice. Furthermore, we examined the mRNA expression levels of BA reabsorption biomarkers in the mouse jejunum and ileum, including Asbt, Ibabp, Osta and Ostb, and found that they were downregulated in response to MMP-12 knockdown (Supplementary Fig. 5d), which is consistent with the reduced serum levels of BAs in these mice. Furthermore, fecal levels of MMP-12 and serum lipids were positively correlated in clinical samples (Fig. 6h).

\section{MMP-12-siRNA-CPA NPs attenuate inflammation and maintain the permeability of mouse small}

intestines. We next explored the inflammatory status in the small intestines of HFD-fed mice treated with MMP-12-siRNA-CPA NPs. As shown in Fig. 7a, HFD feeding significantly decreased the small intestine length, which was reversed by MMP-12 knockdown. Histological analysis indicated that MMP-12-siRNACPA NPs significantly attenuated HFD feeding-induced villous atrophy and crypt hyperplasia (Fig. 7b). Since intestinal goblet cells contribute importantly to intestinal mucin excretion, we therefore examined the numbers of these cells using alcian blue and periodic acid Schiff (AB-PAS) staining. As shown in Fig. $7 \mathrm{~b}, \mathrm{HFD}$ feeding decreased the numbers of goblet cells in the mouse small intestines compared to the normal diet (ND) feeding groups. MMP-12-siRNA-CPA NP treatment restored this reduction. To confirm the severity of intestinal inflammation, we evaluated macrophage (F4/80), neutrophil (Gr-1), and T lymphocyte (CD4) infiltration. Compared with modest changes observed in the ND group, HFD-fed mice displayed increased immune cell infiltration in the small intestines. In contrast, administration of MMP12-siRNA-CPA NPs dramatically blocked these infiltrations (Fig. 7c). Accordingly, the mRNA expression levels of pro-inflammatory cytokines, including $I I-1 \beta$ and Tnf- $a$, were markedly reduced in the small intestines of these mice (Supplementary Fig. 6a). These results indicated that MMP-12 was a critical mediator in HFD feeding-induced small intestinal inflammation.

Small intestinal permeability is affected by inflammation under metabolic stress ${ }^{29}$. We found that HFD feeding induced marked tight junction (TJ) impairments in the mouse small intestinal epithelium, as evidenced by increased serum levels of D-lactitol (D-Lac), impaired TJ structure and reduced protein expression levels of TJ biomarkers, including Occludin and ZO-1. In contrast, MMP-12-siRNA-CPA NP 
intervention significantly attenuated these impairments by restoring them close to the levels of the ND-fed group (Fig. 7d, Supplementary Fig. 6b-d). Of note, the beneficial effects of MMP-12-siRNA-CPA NPs were further confirmed by in vitro studies with increased levels of TJ-associated protein expression and transepithelial electrical resistance (TEER) (Supplementary Fig. 7a-c). Therefore, we speculate that lipids may influence intestinal permeability through the regulation of MMP-12. These results suggested that MMP-12 was a critical factor in mediating the HFD feeding-induced permeability impairments of mouse small intestines.

MMP-12-siRNA-CPA NPs alter the composition of gut microbiota and SCFAs. Gut dysbiosis has been shown to facilitate the development of obesity, whereas obesity further disrupts the composition of gut microbiota, thus forming a vicious circle. ${ }^{18}$ By using bacterial 16S rRNA sequencing (V4 region), a total of $1,648,417$ raw reads were obtained after qualification and were annotated to 699 OTUs. Rarefaction analysis showed that the current sequencing depth covered almost all the gut microbiota within each sample (Supplementary Fig. 8a, b). Unsupervised principal coordinate analysis (PCoA) was used to analyze the whole structural alterations in the gut microbiota among groups. Along the PCo2 axis, MMP12-siRNA-CPA NP treatment reversed HFD feeding-induced dysbiosis, whereas no significant changes were observed in the structure along PCo1 (Supplementary Fig. 8c). Unweighted pair-group method with arithmetic mean (UPGMA) analysis indicated significant separations in the microbiota between ND- and HFD-fed mice and between HFD-fed groups in presence with or without MMP-12-siRNA-CPA NPs, suggesting that MMP-12 knockdown could alter the microbiota composition and shift the microbial community profile from a HFD feeding-induced dysbiotic state towards homeostasis in ND-fed mice (Supplementary Fig. 8d). In addition, taxonomic profiling revealed that MMP-12-siRNA-CPA NP administration markedly reduced the contents of the Proteobacteria phylum in the feces from HFD-fed obese mice (Supplementary Fig. 8e-h). Given that the Proteobacteria phylum is a well-known infective pathogen that contributes to the development of metabolic endotoxemia, MMP-12-siRNA-CPA NPs consistently reduced serum levels of lipopolysaccharide (LPS) in HFD-fed mice (Supplementary Fig. 8i). Such a reduction in LPS could be a critical explanation for the alleviated inflammation in these mice.

Next, we used redundancy analysis (RDA) to clarify the specific bacterial phylotypes changed by HFD signals and MMP-12-siRNA-CPA NP treatment (Supplementary Fig. 8j). Compared to the ND-fed group, HFD signals dramatically altered the 228 OTUs, among which 167 were upregulated and 61 were downregulated. In HFD-fed mice, administration of MMP-12-siRNA-CPA NPs altered 15 OTUs (9 upregulated and 6 downregulated). Interestingly, 6 OTUs were altered by both HFD feeding and MMP-12siRNA-CPA NP administration, and their changes exhibited opposite trends. Among these, OTU_26 and OTU_144 were classified in the detrimental Lachnospiraceae family. This family belongs to the Firmicutes phylum, which is known to aggravate LPS transfer into the circulation system. In addition, 3 OTUs (OTU_30, OTU_1513 and OTU_129) were annotated to the beneficial Eggerthellaceae family, which is a beneficial bacteria responsible for metabolizing secondary plant polyphenols to ameliorate gut inflammation (Fig. 7e, Supplementary Table 4). 
Among gut microbiota-generated metabolites, SCFAs, which are metabolized by intestinal microbiota through indigestible carbohydrates, have been determined to downregulate inflammatory cytokine production in macrophages. We thus evaluated the contents of 6 dominant SCFAs in mouse feces. As shown in Fig. 7f (Supplementary Table 5), HFD signals significantly reduced the contents of SCFAs compared to ND signals. In contrast, such a reduction was reversed by MMP-12-siRNA-CPA NP treatment. Coincident with these results, Spearman correlation analysis revealed that MMP-12-siRNA-CPA NPinduced substantial elevation of SCFAs, including propionic acid, acetic acid and butyric acid, was positively correlated with 3 beneficial OTUs (OTU_30, OTU_1513 and OTU_129) but negatively correlated with 2 detrimental OTUs (OTU_26 and OTU_144) (Fig. 7g, Supplementary Table 6 and 7), suggesting that these OTUs are essential bacteria in MMP-12 knockdown-regulated SCFA production.

Taken together, these data suggested that MMP-12-siRNA-CPA NPs may exert their anti-inflammatory effects and improve gut health by reshaping the gut microbiota and increasing SCFA production.

GM6001-CPA NPs modestly affect HFD feeding-induced obesity. To further dissect whether these beneficial roles of MMP-12 knockdown in mouse small intestines were dependent on its reduced enzymatic activity or expression, we used the CPA NP system carrying the specific MMP-12 inhibitor GM6001 to neutralize its enzymatic activity $(91.73 \pm 1.74 \%$ encapsulation efficiency, Supplementary Fig. 9a). As shown in supplementary Fig. 9b, GM6001 significantly decreased the enzymatic activity of MMP12 in the small intestines of mice fed a HFD. However, GM6001-CPA NPs modestly affected the HFD feeding-induced increase in body weight gain as well as the reduction in food intake (supplementary Fig. 9c, d). In addition, HFD feeding increased blood glucose levels and impaired glucose tolerance, while GM6001-CPA NPs did not alter these parameters. Morphologically, administration of GM6001-CPA NPs modestly affected HFD feeding-induced fasting hyperglycemia, hyperlipidemia, hypercholesterolemia, and glucose intolerance (Supplementary Fig. 9e-g). Similarly, HFD feeding-induced hepatic and epididymal fat accumulation was unaltered in GM6001-CPA NP-treated obese mice (Supplementary Fig. 9h-k). In addition, HFD signals, including increased serum AST and ALT, as well as TG and TC, were similarly unchanged (Supplementary Fig. 9I, m). Taken together, these data revealed that the beneficial role of MMP-12 knockdown was not dependent on its enzymatic function.

FABP4 serves as an effector of MMP-12 in the small intestines of HFD-fed mice. It should be noted that MMP-12 exerts DNA-binding abilities to control gene expression in the nucleus ${ }^{30}$. As shown in Fig. 8a, we found that HFD feeding significantly increased the nuclear and cytosolic contents of MMP-12 proteins in mouse small intestines (jejunum and ileum). Similar results were observed in the in vitro analysis (Fig. $8 b)$. Considering the modest effect of the MMP-12 inhibitor, we speculate that MMP-12 indeed functions as a transcriptional factor involved in the regulation of small intestinal homeostasis. Hence, we performed Spearman correlation analysis to evaluate potential associations among intestinal gene 
expression, gut microbiota composition and SCFA contents. Venn analysis clustered 2 genes, including Fabp4 and Nnmt, which were positive, in line with the above beneficial effects of MMP-12 knockdown (Fig. 8c and Supplementary Table 8-11). We further evaluated the mRNA expression levels of these two genes in response to MMP-12 knockdown in vivo and in vitro and found that Fabp4 mRNA expression was most robustly decreased in the small intestines of HFD-fed mice treated with MMP-12 siRNA NPs and in FFA-treated Caco-2 cells with MMP-12 knockdown (Fig. 8c). The protein expression of FABP4 exhibited a similar tendency (Fig. 8d and Supplementary Fig. 10a-d).

Bioinformatics analysis indicated that a classic MMP-12-binding motif featuring a poly $(A)$ tract of 6 bases was present on the proximal promoter of FABP4 (-148 bp - -142 bp, Fig. 8e). Luciferase activity analysis indicated that overexpression of MMP-12 significantly increased the transcriptional activity of the FABP4 promoter. However, such an activation effect was abolished when this poly $(A)$ tract was mutated (Fig. 8e). Consistently, the mRNA and protein expression levels of FABP4 were correspondingly increased in MMP-12-overexpressing Caco-2 cells (Supplementary Fig. 10e, f). At the epigenetic level, acetyl-histone $\mathrm{H} 3(\mathrm{AcH} 3)$ and $\mathrm{K} 9$-dimethylated histone $\mathrm{H} 3(\mathrm{H} 3 \mathrm{~K} 9-\mathrm{me} 2)$ are two hallmarks associated with chromatin activity and gene transcription ${ }^{31}$. In our study, overexpression of MMP-12 led to robust AcH3 (activation) accumulation, accompanied by a modest impact on the H3K9-me2 levels around the FABP4 proximal promoter (Fig. 8f), suggesting that MMP-12 is a potential enhancer of FABP4 transcription by increasing the accumulation of histone acetylation.

Finally, we wanted to explore whether FABP4 linked MMP-12 signals to the homeostasis of the small intestinal homeostasis. We knocked down FABP4 expression in MMP-12-overexpressing Caco-2 cells. The knockdown efficiency of FABP4 siRNA and the overexpression efficiency of MMP-12 are presented in supplementary Fig. 10g. We found that knockdown of FABP4 partially antagonized MMP-12-facilitated lipid accumulation, pro-inflammatory cytokine expression and secretion, and TJ impairment (Fig. 8g-i and Supplementary Fig. 10g).

\section{Discussion}

MMP-12 is a macrophage metal elastase that cleaves elastin, which is a major component in the media of the small intestines. Macrophages are the major source of MMP-12, the expression of which is markedly increased in various inflammatory diseases ${ }^{25,32}$. Given the causative relationship between inflammation and obesity, MMP-12 expression is increased in mouse WAT in response to HFD-feeding signals. Although MMP-12 is also expressed in the small intestines, whether it contributes to homeostasis and whole-body metabolism remains elusive. In the present study, our findings revealed that HFD signals induced marked MMP-12 expression in mouse small intestines and feces. To specifically and effectively knock down MMP-12 in the small intestines of HFD-fed mice, we successfully constructed an NP system to effectively and orally deliver MMP-12 siRNA. Making the best of this NP system, we found that small intestine-specific MMP-12 knockdown improved HFD feeding-induced metabolic disorders, including hyperglycemia, hyperinsulinemia, glucose intolerance, impaired insulin sensitivity and systemic inflammation. In addition, the HFD-feeding-induced disruptions of lipid uptake/transportation, BA 
reabsorption, permeability and inflammation in mouse small intestines were recuperated by MMP-12 knockdown. We also revealed that small intestinal MMP-12 exerts DNA-binding abilities in the cellular nucleus, rather than its elastolytic activity, when corresponding to HFD-feeding signals, while FABP4 served as its key transcriptional downstream effector. Our findings shed some light on the pathophysiological and transcription-regulatory function of MMP-12 and developed a state-of-the-art siRNA delivery system for gene-specific intervention in mouse small intestines (Fig. 9).

To tissue-specifically regulate MMP-12 expression, we first focused on small intestines and designed a CPA NP system. Given the extensive applications of nanotechnology in delivering various RNAs into tumors $^{33-35}$, herein, we developed a novel NP system for the systemic delivery of siRNA into the small intestines to treat metabolic diseases. Our findings broadened the current applications of the NP system for gene therapies for metabolic disorders. In our present study, CPA NPs exert the following beneficial advantages. For basal materials, CS, isolated from lobsters, crabs and other marine invertebrates, is safe and biocompatible, accompanied by $\mathrm{pH}$-sensitive and adhesive properties ${ }^{36}$. Herein, we established a CS to serve as a protective shelter to avoid gastric acid and to retain the functional siRNA in the small intestines. In addition, as another core component of our NPs, PLGA is a widely used polymer with biodegradable and biocompatible abilities that has been approved in the clinic to deliver antitumor drugs for multiple cancer treatments, including colorectal cancer and ovarian cancer ${ }^{27,37}$. We used this material as a core to coat the PLL-modified siRNAs ${ }^{38}$. By taking advantage of double-emulsion and ionic gelation strategies, we constructed an NP system and effectively applied it for the in vivo knockdown of MMP-12 in mouse small intestines based on the protective effects of CS. Our CPA NP system did not affect MMP12 expression in other organs, including the liver, kidney and colon, demonstrating its excellent specificity in the small intestines. Therefore, the CPA NP system possesses great potential in delivering either RNAs or drugs into the small intestines. Notably, adeno-associated viruses (AAVs) have also been developed to manipulate gene expression in small intestines ${ }^{39}$. However, technical limitations, such as difficulties in the achievement of high-titer AAVs, still restrict the clinical applications of this method ${ }^{40}$. In contrast, our CPA NP system was host-friendly due to its transient impacts on gene expression, since they were prone to be excreted by intestinal peristalsis within the feces. In addition, although MMP inhibition holds significant therapeutic potentials for the treatment of inflammatory diseases, this approach unfortunately has been hindered by significant side effects of MMP inhibitors due to the important physiologic role of MMPs in tissue remodeling ${ }^{41}$. In the present study, we took advantages of the nanosystem to specifically knock down MMP-12 expression in the mouse small intestine and improve the HFD feeding-induced metabolic disorders. This strategy may partially avoid the global side effects caused by MMP inhibitors. Lastly, it does not escape our attention that the small intestines consist of various types of cells, including intestinal villous epithelial cells, columnar cells and goblet cells ${ }^{41}$. Notably, when the mice were fed a HFD, neutrophils and macrophages also infiltrated into the small intestines to trigger inflammatory responses, which facilitated metabolic disorders ${ }^{42,43}$. In our study, we believed that increased MMP-12 expression in mouse small intestines was due to net increased expression levels within all of the above 
cells. Hence, deep single-cell RNA-seq analyses and cell type-specific NP systems based on targeted peptides should be considered for the precision treatment of MMP-12-based metabolic diseases ${ }^{44-46}$.

Considering that MMP-12 is classically identified as an elastase, we blocked MMP-12 enzymatic activity by using the MMP-specific inhibitor GM600147,48. We unexpectedly found that this inhibitor shows modest effects on HFD feeding-induced metabolic physiology, indicating that the beneficial role of MMP12 knockdown did not rely on its enzymatic function. Unprecedentedly, both HFD feeding and FFA stimulation triggered a marked translocation of MMP-12 into the cell nucleus, as evidenced by $\mathrm{IHC}$ and immunocytochemistry (ICC) analyses. These results are in accordance with a previous study, which suggested that MMP-12 also exerts DNA-binding abilities ${ }^{30}$ and thus functions as a transcriptional factor in response to virus infection. Similarly, in mouse small intestines, MMP-12 activates FABP4 transcription by directly binding to the poly $(A)$ tract presented on its proximal promoter. Histones respond sensitively to external signals, such as food and light, and thus undergo multiple posttranslational modifications, including acetylation and methylation ${ }^{49,50}$. However, the MMP-12-triggered epigenetic regulation of histones remains elusive. Our findings showed that overexpression of MMP-12 transformed the local chromatin environment of the poly $(A)$ tract on the FABP4 promoter into an active state, which was confirmed by increased $\mathrm{AcH} 3$ (an active biomarker) accumulation. However, we found that the levels of H3K9-me2 were not altered by MMP-12 overexpression. These results indicated that MMP-12 may function as an enhancer to facilitate gene transcription by altering histone acetylation rather than methylation. Hence, our findings extend the current recognition of the epigenetic regulation of lipid transportation and intestinal homeostasis via the MMP-12/FABP4 axis. Additionally, since the FABP4 was demonstrated as the MMP-12 downstream effector, it might function as a more specific and safer therapeutic target instead of MMP-12 and minimize the MMP-12 inactivity-induced side effects in future studies.

In conclusion, the present study highlights a nonclassic transcriptional regulatory function of MMP-12 in mouse small intestines that activates Fabp4 transcription to regulate the homeostasis of small intestinal homeostasis and whole-body metabolism. In addition, we established a small intestine-specific CPA NP system, thus providing proof-of-concept siRNA-based metabolic gene manipulations. Translational research that advances the clinical applications of the CPA NP system and therapeutic interventions targeting MMP-12 will benefit patients with obesity and its associated diseases.

\section{Methods}

Animals. Male C57BL/6J mice were purchased from the Model Animal Research Center of Nanjing University (Nanjing, Jiangsu, China). Male C57BL/6J mice were maintained in a $12 \mathrm{~h}$ LD cycle and in a temperature- and humidity-controlled environment. Note that we housed these mice at a thermoneutral temperature $\left(26 \pm 1^{\circ} \mathrm{C}\right)$ to avoid the mild cold $\left(22 \pm 1^{\circ} \mathrm{C}\right)$-induced activation impacts on the energy expenditure of mice. All experiments were approved and conducted according to the guidance of the Laboratory Animal Care \& Use Committee at China Pharmaceutical University (Permit number SYXK2016-0011). To establish the HFD-fed obese model, 10-week-old mice were fed with HFD (60\% kcal from 
fat, Research Diets, New Brunswick, NJ, USA) for 11 weeks. To dissect the role of MMP-12 in the regulation of intestinal homeostasis, MMP-12 expression was knocked down in the mouse small intestine by using CPA NPs carrying either a MMP-12 siRNA mixture (at a dose of $40 \mu \mathrm{g} / \mathrm{kg}$ body weight once every 3 days) or its corresponding control for a total of 27 days. The following day after final gavage, mice were analyzed and later sacrificed to collect sera, tissues and feces for further analyses.

Statistical analysis. Statistical analysis was performed by using the GraphPad Prism 7 programme (California, USA). Groups of data were expressed as the mean \pm standard deviation (SD). One-way analysis of variance (ANOVA) followed by Tukey's multiple comparisons test. was performed to analyze the data. Spearman correlation analysis was performed between short chain fatty acids (SCFAs), gene expression, and operational taxonomic units (OTUs) using psych package of $\mathrm{R}$, and the correlation heatmap was drawn through the heatmap package. A $p$ value $<0.05$ was considered statistically significant.

\section{References}

1. Swinburn, B.A., et al. The Global Syndemic of Obesity, Undernutrition, and Climate Change: The Lancet Commission report. The Lancet 393, 791-846 (2019).

2. Maresch, L.K., et al. Intestine-Specific Overexpression of Carboxylesterase 2c Protects Mice From Diet-Induced Liver Steatosis and Obesity. Hepatol Commun 3, 227-245 (2019).

3. Upadhyay, J., Farr, O., Perakakis, N., Ghaly, W. \& Mantzoros, C. Obesity as a Disease. Med Clin North Am 102, 13-33 (2018).

4. Ko, C.W., Qu, J., Black, D.D. \& Tso, P. Regulation of intestinal lipid metabolism: current concepts and relevance to disease. Nat Rev Gastroenterol Hepatol 17, 169-183 (2020).

5. Xu, Y., et al. Novel strategy for oral peptide delivery in incretin-based diabetes treatment. Gut $69,911-$ 919 (2020).

6. Wan, Y., et al. Effects of dietary fat on gut microbiota and faecal metabolites, and their relationship with cardiometabolic risk factors: a 6-month randomised controlled-feeding trial. Gut 68, 1417-1429 (2019).

7. Kuipers, F., Bloks, V.W. \& Groen, A.K. Beyond intestinal soap-bile acids in metabolic control. Nat Rev Endocrinol 10, 488-498 (2014).

8. Alexopoulos, S.J., et al. Mitochondrial uncoupler BAM15 reverses diet-induced obesity and insulin resistance in mice. Nat Commun 11, 2397 (2020).

9. Shi, S., et al. Drug Delivery Strategies for the Treatment of Metabolic Diseases. Adv Healthc Mater 8 , e1801655 (2019).

10. Zhang, Y., Sowers, J.R. \& Ren, J. Targeting autophagy in obesity: from pathophysiology to management. Nat Rev Endocrino/ 14, 356-376 (2018). 
11. Wang, W., et al. Tauroursodeoxycholic acid inhibits intestinal inflammation and barrier disruption in mice with non-alcoholic fatty liver disease. Br J Pharmacol 175, 469-484 (2018).

12. Luo, H., et al. AIDA Selectively Mediates Downregulation of Fat Synthesis Enzymes by ERAD to Retard Intestinal Fat Absorption and Prevent Obesity. Cell Metab 27, 843-853 e846 (2018).

13. Hersoug, L.G., Moller, P. \& Loft, S. Gut microbiota-derived lipopolysaccharide uptake and trafficking to adipose tissue: implications for inflammation and obesity. Obes Rev 17, 297-312 (2016).

14. Zhang, F., et al. Lacteal junction zippering protects against diet-induced obesity. Science $\mathbf{3 6 1}$, 599603 (2018).

15. Xiao, C., Stahel, P., Carreiro, A.L., Buhman, K.K. \& Lewis, G.F. Recent Advances in Triacylglycerol Mobilization by the Gut. Trends Endocrinol Metab 29, 151-163 (2018).

16. Wang, B., et al. Intestinal Phospholipid Remodeling Is Required for Dietary-Lipid Uptake and Survival on a High-Fat Diet. Cell Metab 23, 492-504 (2016).

17. Virtue, A.T., et al. The gut microbiota regulates white adipose tissue inflammation and obesity via a family of microRNAs. Sci Trans/ Med 11(2019).

18. Martinez-Guryn, K., et al. Small Intestine Microbiota Regulate Host Digestive and Absorptive Adaptive Responses to Dietary Lipids. Cell Host Microbe 23, 458-469 e455 (2018).

19. Garidou, L., et al. The Gut Microbiota Regulates Intestinal CD4 T Cells Expressing RORyt and Controls Metabolic Disease. Cell Metabolism 22, 100-112 (2015).

20. Saltiel, A.R. \& Olefsky, J.M. Inflammatory mechanisms linking obesity and metabolic disease. J Clin Invest 127, 1-4 (2017).

21. Campbell, C., et al. Bacterial metabolism of bile acids promotes generation of peripheral regulatory $T$ cells. Nature 581, 475-479 (2020).

22. Hall, J.E., do Carmo, J.M., da Silva, A.A., Wang, Z. \& Hall, M.E. Obesity, kidney dysfunction and hypertension: mechanistic links. Nat Rev Nephrol 15, 367-385 (2019).

23. Kim, J.H., et al. Regulation of the catabolic cascade in osteoarthritis by the zinc-ZIP8-MTF1 axis. Cell 156, 730-743 (2014).

24. Shan, X., Tomlinson, L., Yang, Q. \& Colognato, H. Distinct Requirements for Extracellular and Intracellular MMP12 in the Development of the Adult V-SVZ Neural Stem Cell Niche. Stem Cell Reports 10, 984-999 (2018).

25. Koshman, Y.E., et al. Regulation of connective tissue growth factor gene expression and fibrosis in human heart failure. J Card Fail 19, 283-294 (2013).

26. Yan, W.J., et al. Corn fiber gum-soybean protein isolate double network hydrogel as oral delivery vehicles for thermosensitive bioactive compounds. Food Hydrocolloid 107(2020).

27. Islam, M.A., et al. Restoration of tumour-growth suppression in vivo via systemic nanoparticlemediated delivery of PTEN mRNA. Nat Biomed Eng 2, 850-864 (2018).

28. Xiang, S., Sarem, M., Shah, S. \& Shastri, V.P. Liposomal Treatment of Cancer Cells Modulates Uptake Pathway of Polymeric Nanoparticles by Altering Membrane Stiffness. Small 14(2018). 
29. Monteiro-Sepulveda, M., et al. Jejunal T Cell Inflammation in Human Obesity Correlates with Decreased Enterocyte Insulin Signaling. Cell Metab 22, 113-124 (2015).

30. Marchant, D.J., et al. A new transcriptional role for matrix metalloproteinase-12 in antiviral immunity. Nat Med 20, 493-502 (2014).

31. Zhang, W., et al. SWI/SNF complex subunit BAF60a represses hepatic ureagenesis through a crosstalk between YB-1 and PGC-1 alpha. Mol Metab 32, 85-96 (2020).

32. Lattanzi, S., Di Napoli, M., Ricci, S. \& Divani, A.A. Matrix Metalloproteinases in Acute Intracerebral Hemorrhage. Neurotherapeutics 17, 484-496 (2020).

33. Ren, Y., et al. Targeted tumor-penetrating siRNA nanocomplexes for credentialing the ovarian cancer oncogene ID4. Sci Transl Med 4, 147ra112 (2012).

34. Choi, K.Y., et al. Binary Targeting of siRNA to Hematologic Cancer Cells In Vivo using Layer-by-Layer Nanoparticles. Adv Funct Mater 29(2019).

35. Kanasty, R., Dorkin, J.R., Vegas, A. \& Anderson, D. Delivery materials for siRNA therapeutics. Nat Mater 12, 967-977 (2013).

36. Guo, H.H., et al. Dual-Stimuli-Responsive Gut Microbiota-Targeting Berberine-CS/PT-NPs Improved Metabolic Status in Obese Hamsters. Advanced Functional Materials 29(2019).

37. Xue, Y., Xu, X., Zhang, X.Q., Farokhzad, O.C. \& Langer, R. Preventing diet-induced obesity in mice by adipose tissue transformation and angiogenesis using targeted nanoparticles. Proc Natl Acad Sci U $S A 113,5552-5557$ (2016).

38. Risnayanti, C., Jang, Y.S., Lee, J. \& Ahn, H.J. PLGA nanoparticles co-delivering MDR1 and BCL2 siRNA for overcoming resistance of paclitaxel and cisplatin in recurrent or advanced ovarian cancer. Sci Rep 8, 7498 (2018).

39. He, J., et al. Fbxw7 increases CCL2/7 in CX3CR1hi macrophages to promote intestinal inflammation. 129, 3877-3893 (2019).

40. Smith, R., et al. Rituximab as therapy to induce remission after relapse in ANCA-associated vasculitis. (2020).

41. Lavery, D.L., et al. Evolution of oesophageal adenocarcinoma from metaplastic columnar epithelium without goblet cells in Barrett's oesophagus. Gut 65, 907-913 (2016).

42. Garrett, W.S., Gordon, J.I. \& Glimcher, L.H. Homeostasis and inflammation in the intestine. Ce// 140, 859-870 (2010).

43. Fuke, N., Nagata, N., Suganuma, H. \& Ota, T. Regulation of Gut Microbiota and Metabolic Endotoxemia with Dietary Factors. Nutrients 11(2019).

44. Li, Q., et al. Developmental Heterogeneity of Microglia and Brain Myeloid Cells Revealed by Deep Single-Cell RNA Sequencing. 101, 207-223.e210 (2019).

45. Biase, F., Cao, X. \& Zhong, S.J.G.r. Cell fate inclination within 2-cell and 4-cell mouse embryos revealed by single-cell RNA sequencing. 24, 1787-1796 (2014). 
46. Ni, Q., et al. A bi-adjuvant nanovaccine that potentiates immunogenicity of neoantigen for combination immunotherapy of colorectal cancer. 6, eaaw6071 (2020).

47. Meissburger, B., Stachorski, L., Roder, E., Rudofsky, G. \& Wolfrum, C. Tissue inhibitor of matrix metalloproteinase 1 (TIMP1) controls adipogenesis in obesity in mice and in humans. Diabetologia 54, 1468-1479 (2011).

48. Strong, A.L., et al. Obesity-associated dysregulation of calpastatin and MMP-15 in adipose-derived stromal cells results in their enhanced invasion. Stem Cells 30, 2774-2783 (2012).

49. Berger, S.L. An embarrassment of niches: the many covalent modifications of histones in transcriptional regulation. Oncogene 20, 3007-3013 (2001).

50. Strahl, B.D. \& Allis, C.D. The language of covalent histone modifications. Nature 403, 41-45 (2000).

\section{Declarations}

\section{Acknowledgements}

This work was financially supported by grants from the National Natural Science Foundation of China (31800992 to SYC, 31771298 to CL, 81800512 to WXZ), the Natural Science Foundation of Jiangsu Province (BK20180554 to SYC, BK20180577 to WXZ), the Project of State Key Laboratory of Natural Medicines, China Pharmaceutical University (SKLNMZZ202005 to CL), the “Double First-Class" University Project (CPU2018GY17 and CPU2018GY18 to CL), and the Priority Academic Program Development of Jiangsu Higher Education Institutions (PAPD, to CL and SYC).

\section{Author contributions}

Conception of the study: Mingming Song, Chang Liu and Siyu Chen; design of the study protocol: Mingming Song, Zixuan Tao, literature search and data extraction: Yujie Shi and Yonghong Xiong, Wenxiang Zhang, Jianning Li; statistical analysis: Mingming Song, Shiyao Zhang; manuscript draft: Mingming Song, Siyu Chen.

\section{Conflict of interest:}

All authors declare no conflict of interest.

\section{Figures}



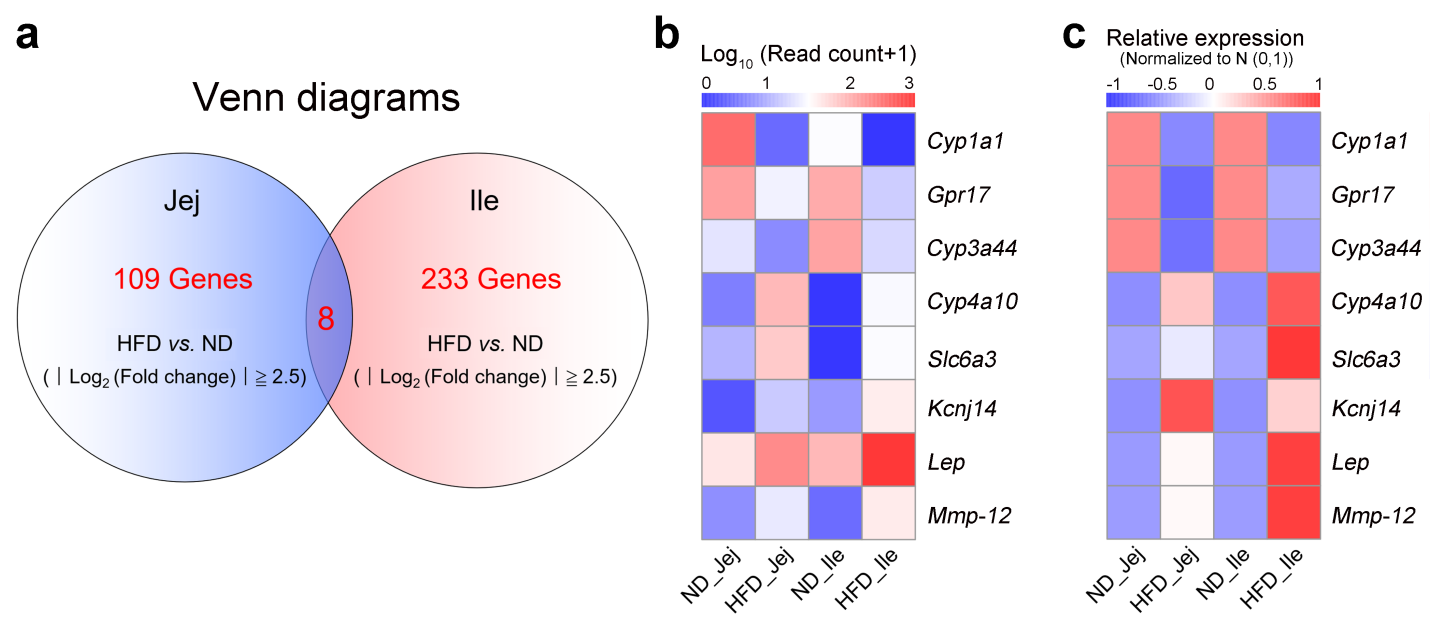

d

e
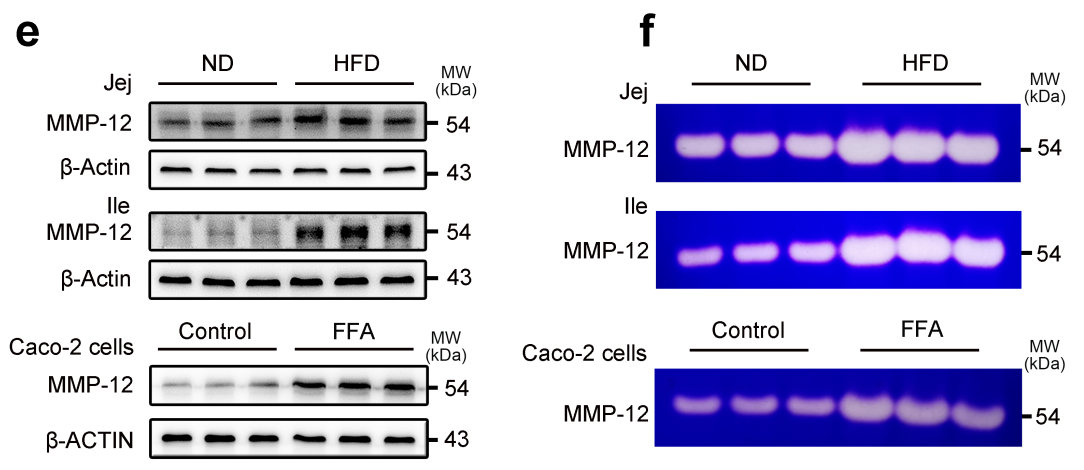

g

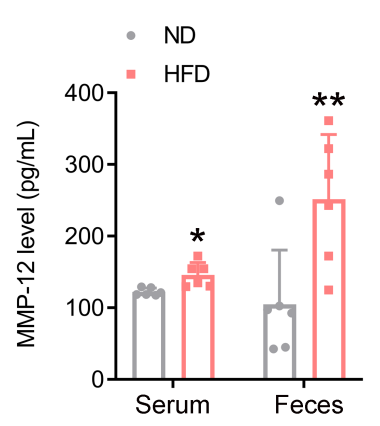

- Control

- FFA

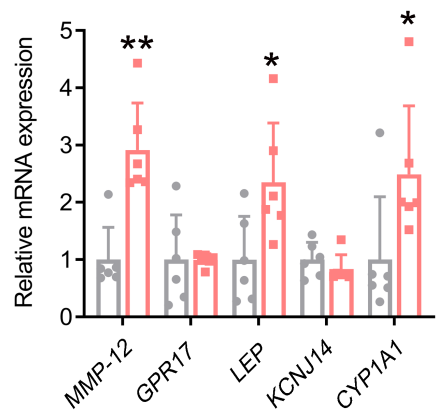

h

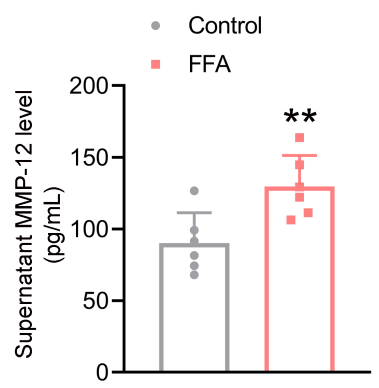

\section{Figure 1}

MMP-12 responses to HFD signals in mouse small intestines. (a) Venn diagrams of high-throughput RNA sequencing results from small intestinal samples (including jejunum and ileum, respectively) of mice fed either an ND or an HFD for 11 weeks. (b) Heat map of eight clustered genes derived from high-throughput RNA sequencing results. (c) RT-qPCR validation of eight clustered gene mRNA expression levels in the small intestine of HFD-fed mice. $n=6$ for each group. (d) RT-qPCR analyses of mRNA expression levels of 5 genes in Caco- 2 cells treated with 0.4 mM FFAs for $24 \mathrm{~h}$. ${ }^{\star \star} \mathrm{p}<0.01$ vs. control group. $n=6$ for each group. (e) Western blot analysis of MMP-12 protein expression levels in the small intestines of HFD-fed mice (upper) and FFA-treated Caco-2 cells (below). (f) Zymographic analysis of MMP-12 enzymatic activity in the small intestines of HFD-fed mice (upper) and FFA-treated Caco-2 cells (below). (g) Serum and fecal MMP-12 levels in HFD-fed mice. ${ }^{*} p<0.05,{ }^{*} p<0.01$ vs. ND group. $n=6$ for each group. (h) Supernatant MMP-12 levels in FFA-treated Caco-2 cells. $n=6$ for each group. ${ }^{*} p<0.05,{ }^{\star *} p<0.01$ vs. Control group. All data are presented as the mean \pm SD. One-way ANOVA followed by Tukey's multiple comparisons test. Jej: jejunum; lle: ileum. 
a

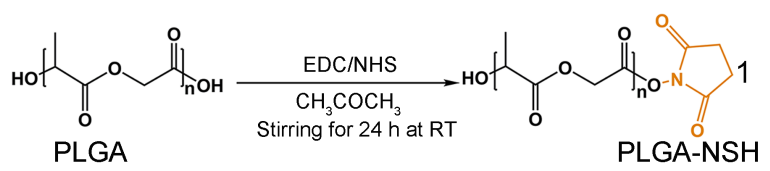<smiles>CCCCC(=O)OCC(=O)ON1C(=O)CCC1=O</smiles>

b

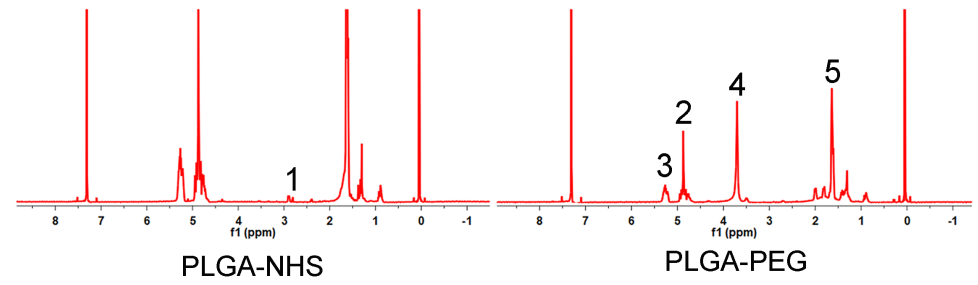

C

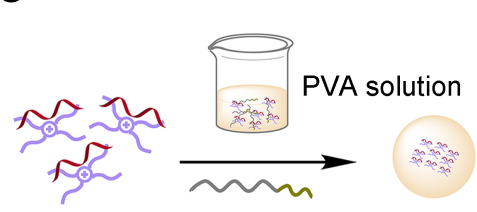

siRNA/PLL PLGA-PEG siRNA/PLGA NPs CS
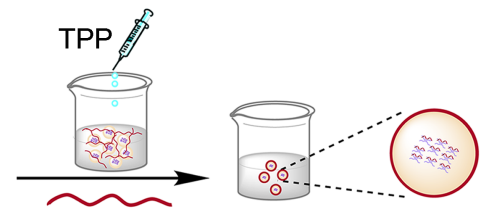
siRNA/CPA NPs d

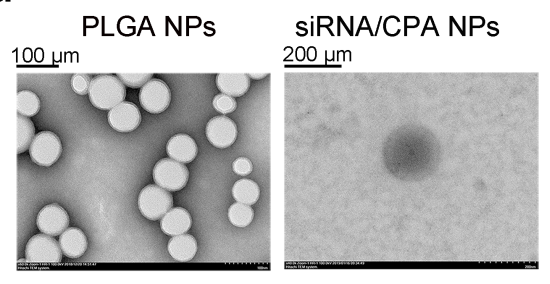

SiRNA/CPA NPs

e

\section{f}

\begin{tabular}{cccc}
\hline Nanoparticles & Average size $(\mathrm{nm})$ & PDI & $\zeta$-potential $(\mathrm{mV})$ \\
\hline PLGA NPs & $158.4 \pm 8.36$ & $0.075 \pm 0.02$ & $-14.1 \pm 4.97$ \\
siRNA/CPA NPs & $226.9 \pm 0.70$ & $0.23 \pm 0.01$ & $57.8 \pm 4.98$ \\
\hline
\end{tabular}

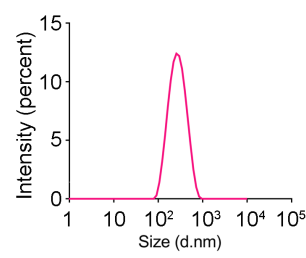

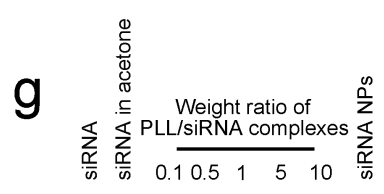

- Reaction medium

$=$ SGF

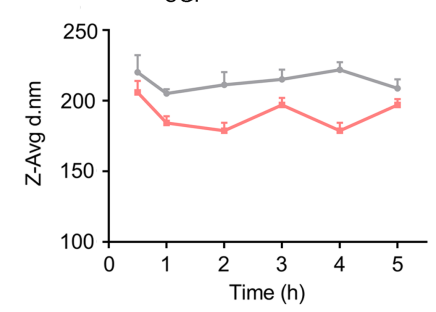

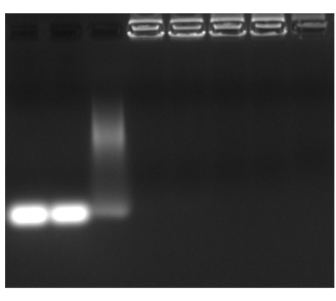

\section{Figure 2}

Preparation and Characterization of CPA NPs. (a) The reaction scheme of PLGA-PEG synthesis. (b) $1 \mathrm{H}$ NMR spectra of PLGA-PEG. (c) Synthesis process of CPA NPs and the schematic representation of particle structure. (d) Representative TEM images of PLGA NPs (scale bars: $100 \mu \mathrm{m}$ ) and CPA NPs (left) (scale bars: $100 \mu \mathrm{m}$ ), size distribution of CPA NPs measured by Mastersizer Micro (right). (e) Zetapotential of PLGA NPs and CPA NPs. (f) Stability of CPA NPs in the simulated gastric fluid (SGF) for the indicated times, $n=3$ for each group. All values are presented as the mean \pm SD. (g) Agarose gel electrophoresis assay of siRNA stability in organic solvent, naked or complexed with PLL. 
a

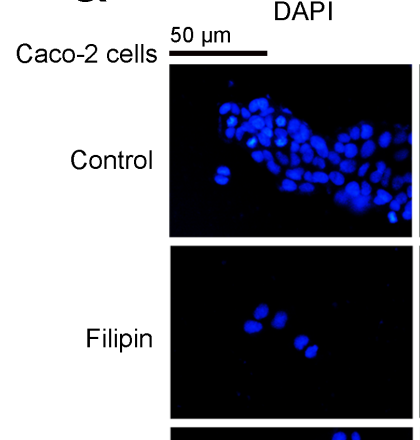

CPZ

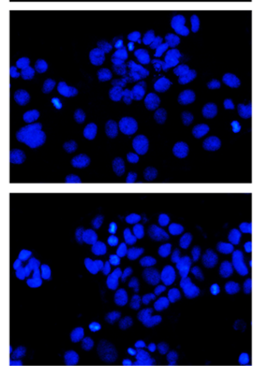

b

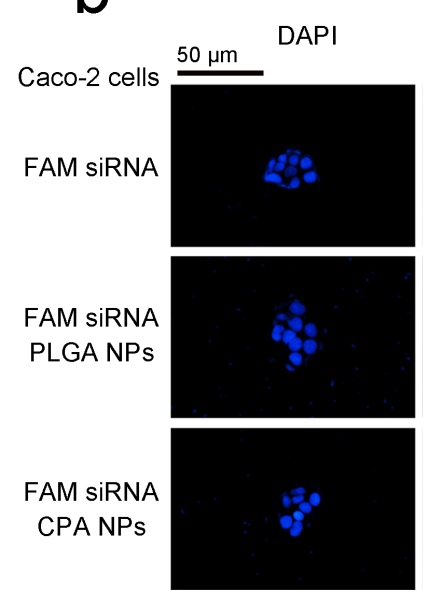

C

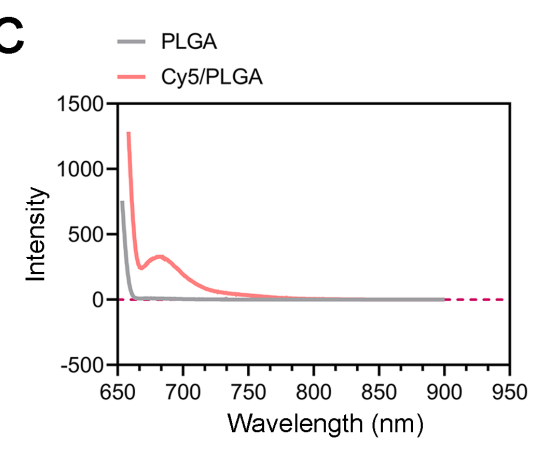

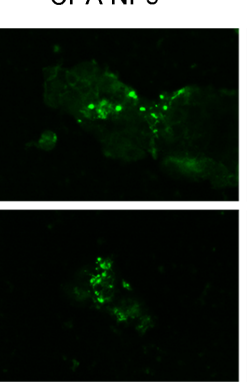
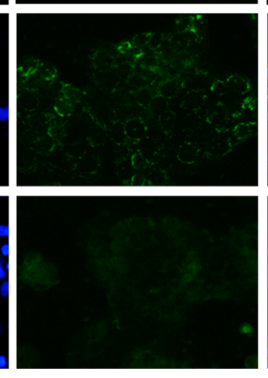

Merge
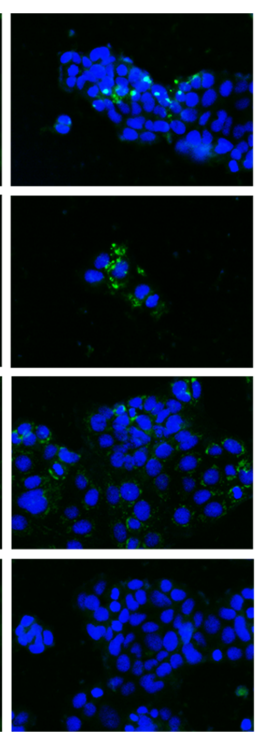

FAM siRNA
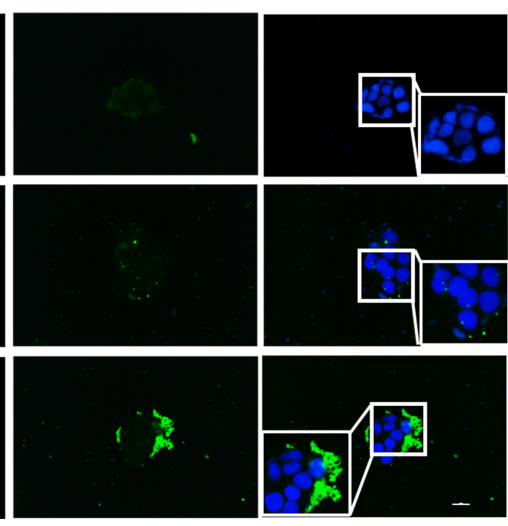

d

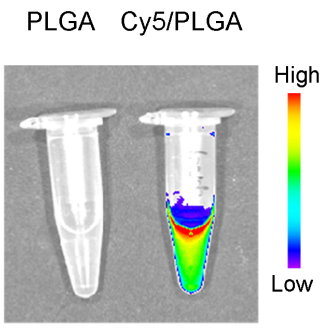

e

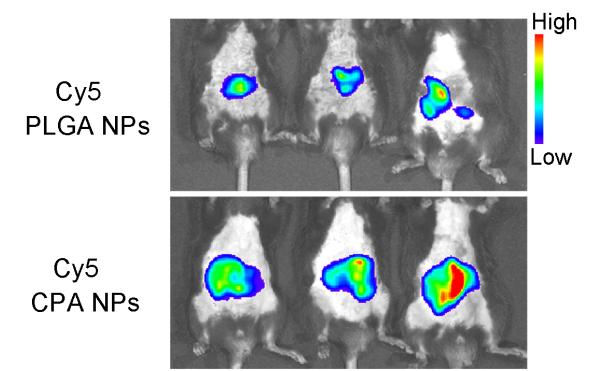

$f$

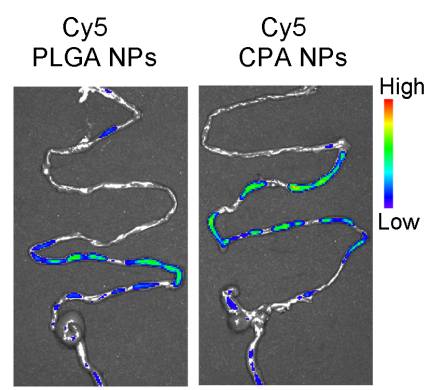

g

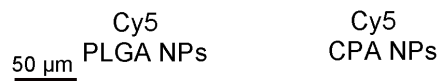

DAPI

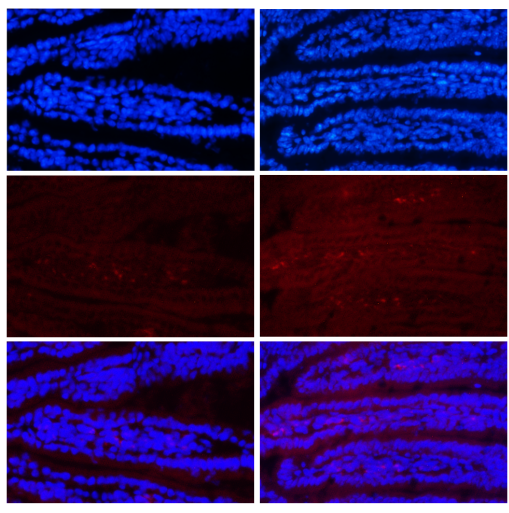

$\mathrm{h}$

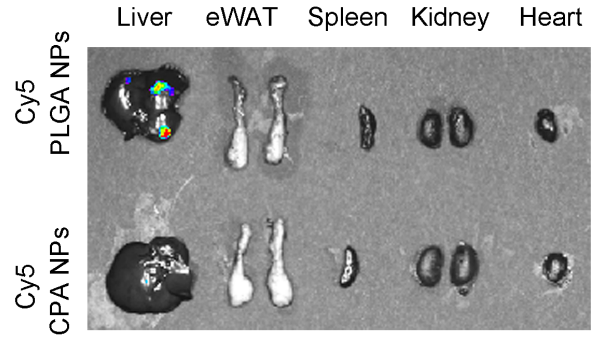

\section{Figure 3}

Mechanism of cellular uptake and biodistribution of CPA NPs in vitro and in vivo. (a) Representative fluorescence microscopy images of Caco-2 cells treated with either FAM siRNA CPA NPs alone or in combination with indicated inhibitors (scale bars: $50 \mu \mathrm{m}$ ). (b) Fluorescence microscopy images of Caco-2 cells transfected with FAM siRNA/PLGA NPs or FAM siRNA/CPA NPs (scale bars: $50 \mu \mathrm{m}$ ). (c, d) UV-Vis spectroscopy (left) and photofluorogram (right) confirming successful synthesis of Cy5/PLGA by 
measuring the absorbance at $680 \mathrm{~nm}$. (e) Distribution of the indicated NPs in vivo. (f) Representative images of the intestinal tract of mice $48 \mathrm{~h}$ after administration of Cy5/PLGA NPs and Cy5/CPA NPs by intragastric administration. (g) Histological sections from the intestine $48 \mathrm{~h}$ after oral administration of Cy5/PLGA NPs and Cy5/CPA NPs (scale bars: $50 \mu \mathrm{m}$ ). (h) Biodistribution of Cy5/PLGA NPs and Cy5/CPA NPs in the mouse liver, eWAT, spleen, kidney and heart. All values are presented as the mean \pm SD.

a
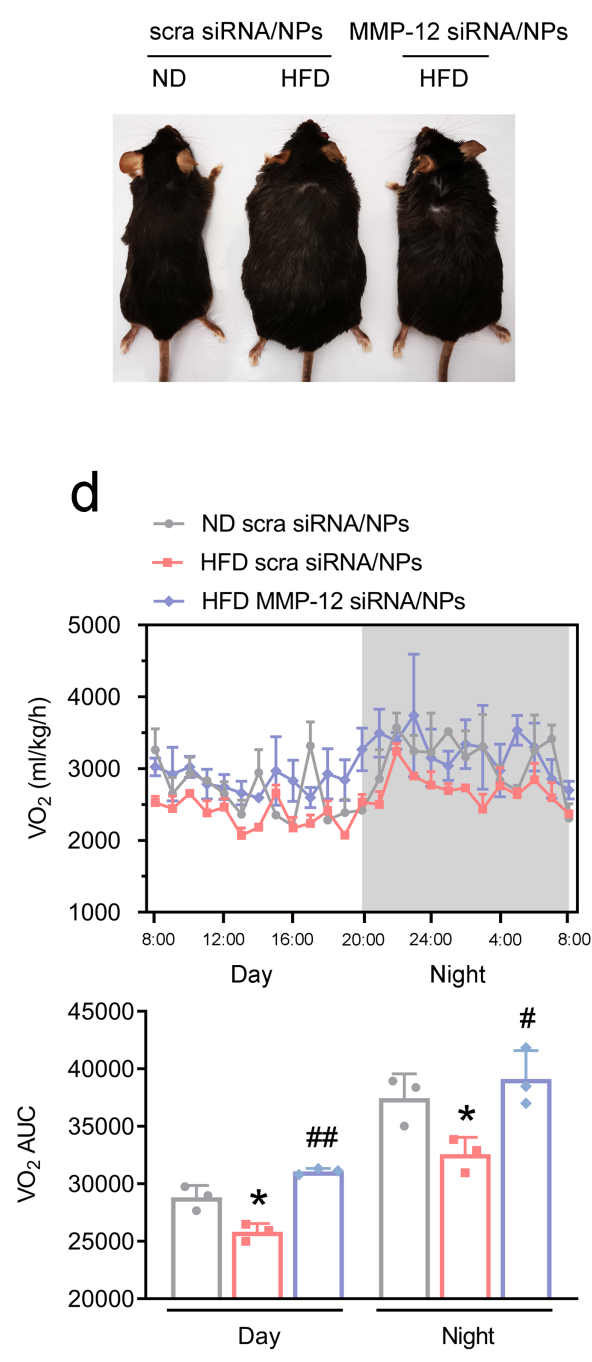

g

- ND scra siRNA/NPs

- HFD scra siRNA/NPs

- HFD MMP-12 siRNA/NPs

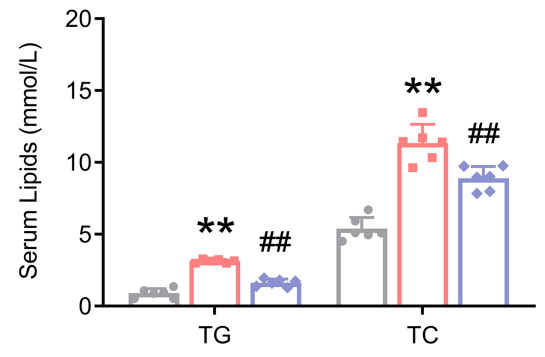

b

$\rightarrow$ ND scra siRNA/NPs

- HFD scra siRNA/NPs

$\rightarrow$ HFD MMP-12 siRNA/NPs

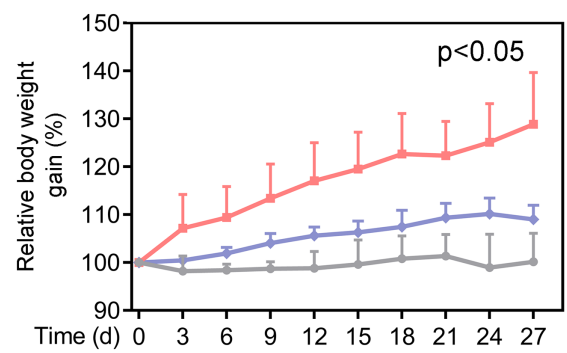

e
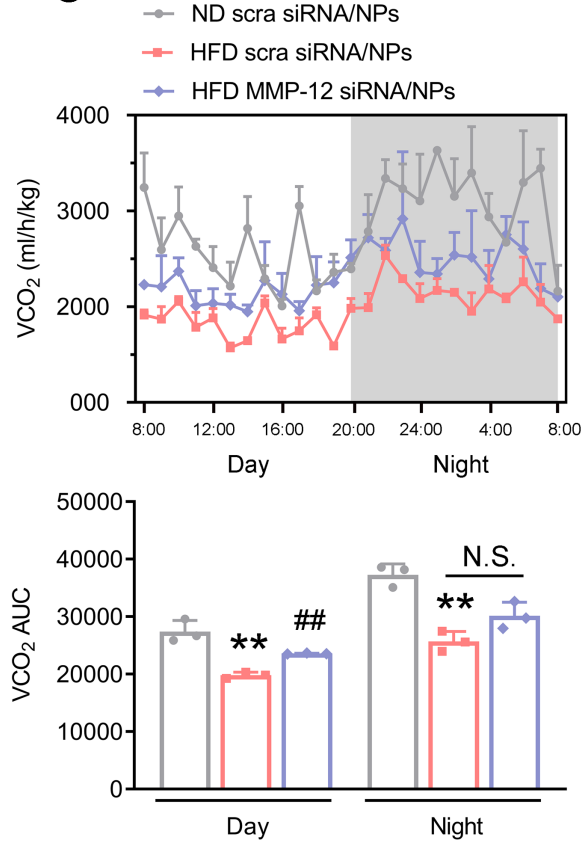

h

$\rightarrow$ ND scra siRNA/NPs

- HFD scra siRNA/NPs

$\rightarrow$ HFD MMP-12 siRNA/NPs

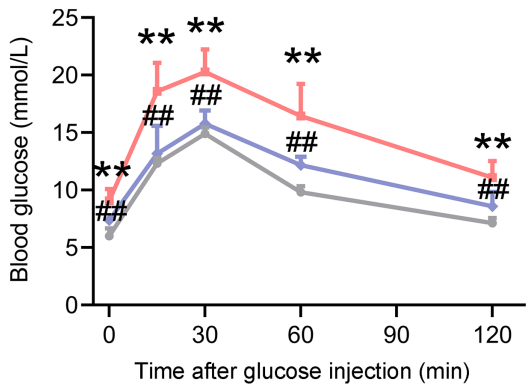

C

$\begin{array}{ll}\text { - } & \text { ND scra siRNA/NPs } \\ \text { - HFD scra siRNA/NPs } \\ \text { - HFD MMP-12 siRNA/NPs }\end{array}$

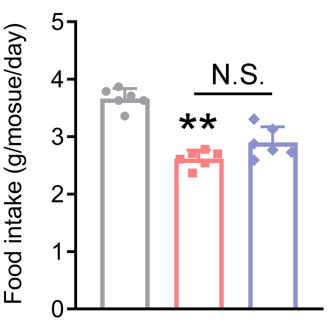

f

- ND scra siRNA/NPs

- HFD scra siRNA/NPs
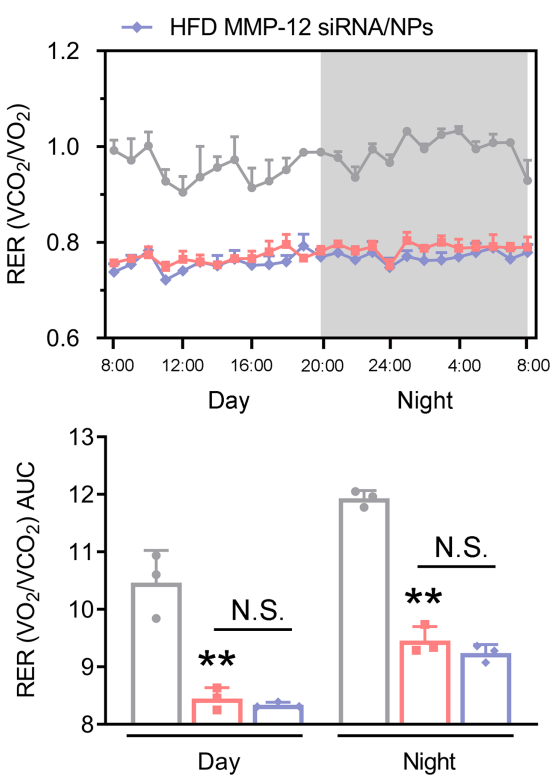

i

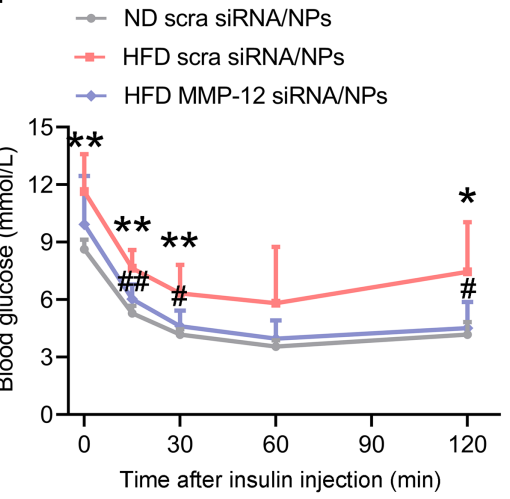

Figure 4 
MMP-12-siRNA-CPA NPs improve HFD feeding-induced obesity and insulin resistance in mice. ND and HFD mice were treated with either MMP-12-siRNA NPs or scra-siRNA NPs every 3 days for a total of 27 days through intragastric administration. (a) Representative mice were photographed at the end of the experiment. (b) Relative body weight gain. (c) Food intake. For (a-c), n=6 for each group. (d-f) VO2, VCO2 and respiration exchange ratio (RER) (VCO2/VO2) were assessed by metabolic cage analysis. $n=3$ for each group. (g) Serum lipid levels. (h, i) Glucose tolerance test (GTT) and insulin tolerance test (ITT) analyses. $\mathrm{n}=6$ for each group. ${ }^{\star} \mathrm{p}<0.05,{ }^{\star \star} \mathrm{p}<0.01 \mathrm{vs.}$. ND scra siRNA/NP group, $\# p<0.05, \# \# p<0.01$ vs. HFD MMP-12 siRNA/NP group, N.S.: no significance. All data are presented as the mean \pm SD. One-way ANOVA followed by Tukey's multiple comparisons test. Jej: jejunum; lle: ileum.

a

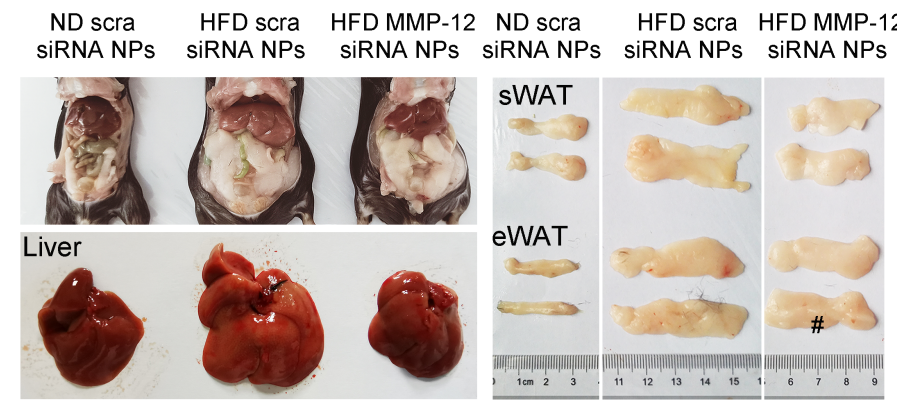

b

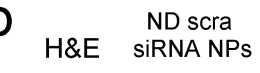

$\stackrel{亠}{\perp}$

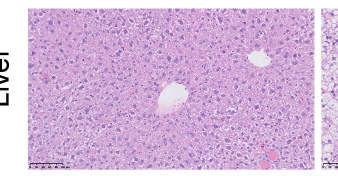

放

放
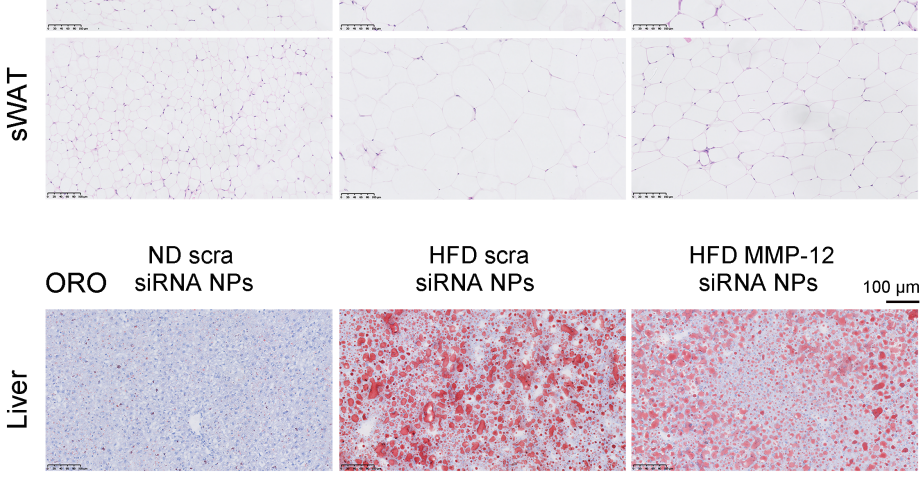

C

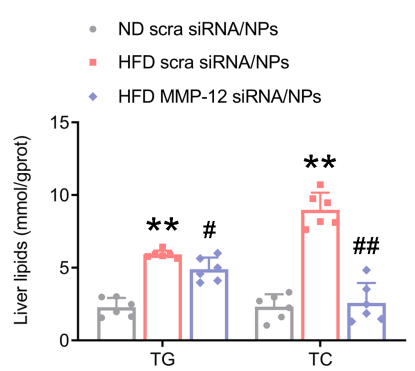

e SIRNA NPS

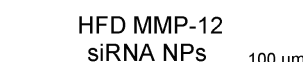

於

f
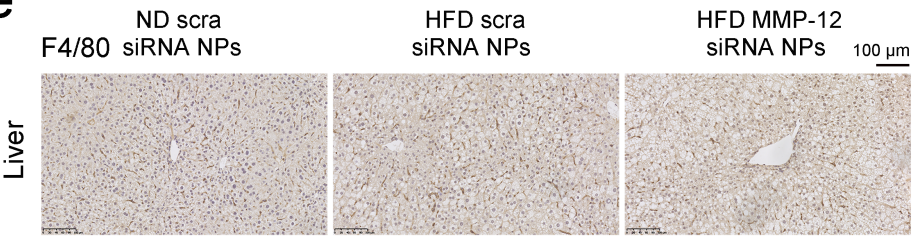

年

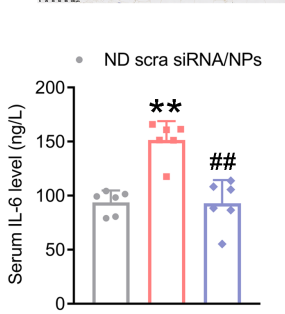

d

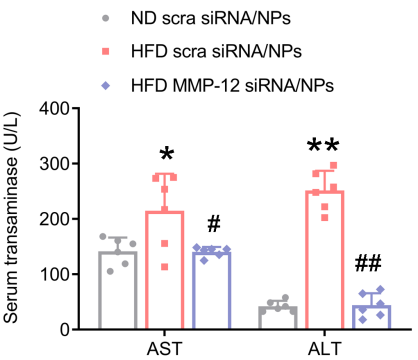

HFD MMP-12 RNA NPS $100 \mu \mathrm{m}$

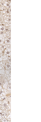
然
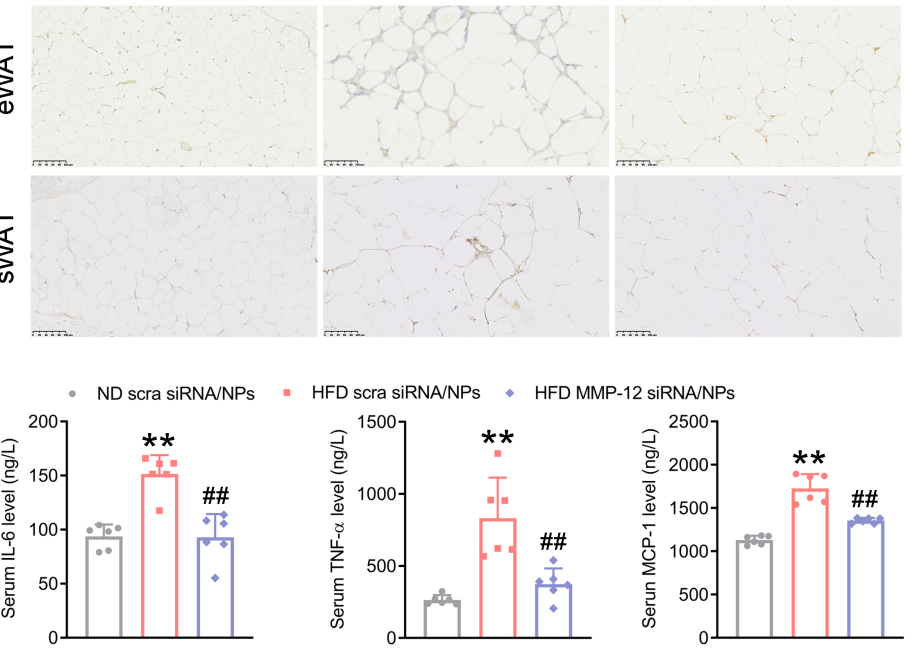

\section{Figure 5}

MMP-12-siRNA-CPA NPs attenuate inflammation and maintain the permeability of mouse small intestines. (a) Morphologic photos of humanely killed mice (left, upper); Representative images of liver (left, below), eWAT and sWAT (right). (b) Representative images of hematoxylin and eosin (H\&E) staining for liver, eWAT and sWAT sections (upper) and oil red O (ORO) staining for liver lipid accumulation (below) (scale bars: $100 \mu \mathrm{m}$ ). (c) Liver TG and TC contents. (d) Serum transaminases. (e) IHC analysis of F4/80 expression in the liver, eWAT and sWAT (scale bars: $100 \mu \mathrm{m}$ ). (f) Serum IL-6, MCP-1 and TNF-a 
levels. $\mathrm{n}=6$ for each group. ${ }^{\star} \mathrm{p}<0.05,{ }^{\star \star} \mathrm{p}<0.01$ vs. ND scra siRNA/NP group, $\# p<0.05, \# \# p<0.01$ vs. HFD MMP-12 siRNA/NP group. All data are presented as the mean \pm SD. One-way ANOVA followed by Tukey's multiple comparisons test.
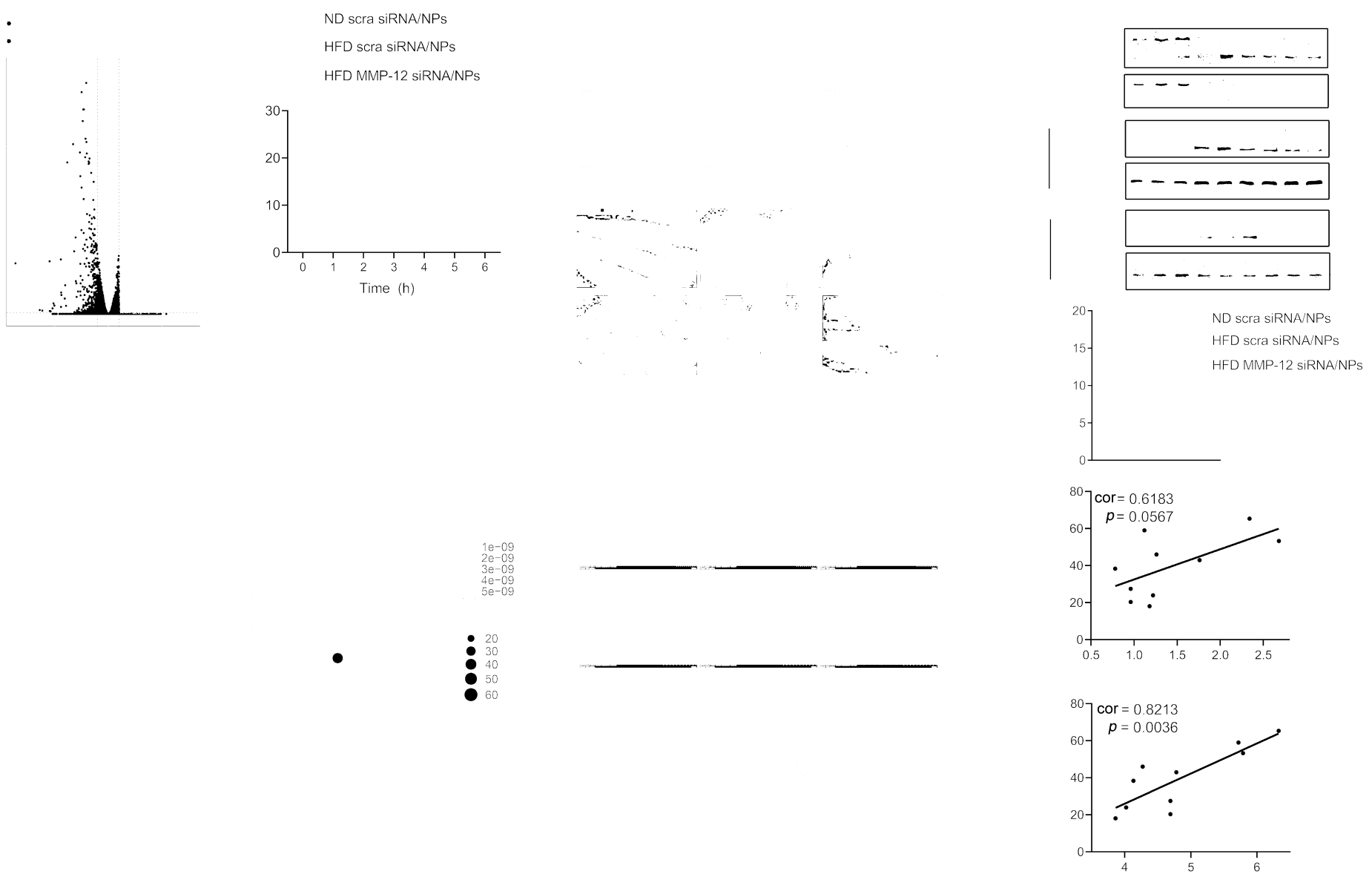

\section{Figure 6}

MMP-12-siRNA-CPA NPs improve lipid transportation and BA reabsorption in the small intestines of HFDfed mice. (a) Genome-wide changes in mRNA expression shown in a volcano plot. The number of genes up- or downregulated by 2 -fold or more with $p<0.05$. (b) GO analysis of biological pathways using the ClusterProfiler package of R software. (c) Postprandial TG response in mice after oral gavage with olive oil (10 $\mu \mathrm{L}$ /body weight), $\mathrm{n}=6$ for each group. ${ }^{*} \mathrm{p}<0.05,{ }^{\star \star} \mathrm{p}<0.01$ vs. ND scra siRNA/NP group, $\# \mathrm{p}<0.05$, $\# \# p<0.01$ vs. HFD MMP-12 siRNA/NP group. One-way ANOVA followed by Tukey's multiple comparisons test. (d) H\&E and ORO staining of intestine sections from three different groups of mice after oral gavage with olive oil for $2 \mathrm{~h}$. (scale bars: $200 \mu \mathrm{m}$ ). (e) Serum chylomicron particle size in mice (upper) and a representative picture of serum collected from mice (below) (scale bars: $200 \mu \mathrm{m}$ ). (f) Western blot analysis of ApoB-48 expression in mouse serum, chylomicron, jejunum and ileum. (g) Serum total BA (TBA) levels. For a-g, $n=6$ for each group. ${ }^{*} p<0.05, * \star p<0.01$ vs. ND scra siRNA/NP group, $\# p<0.05$, $\# \# p<0.01$ vs. HFD MMP-12 siRNA/NP group. All data are presented as the mean \pm SD. (h) Correlation of 
serum TG and TC levels with fecal MMP-12 levels in human subjects. $n=10$. The correlation coefficients ( $r$ value) and $p$ values were calculated by Pearson analysis. CM: chylomicron; Jej: jejunum; lle: ileum.
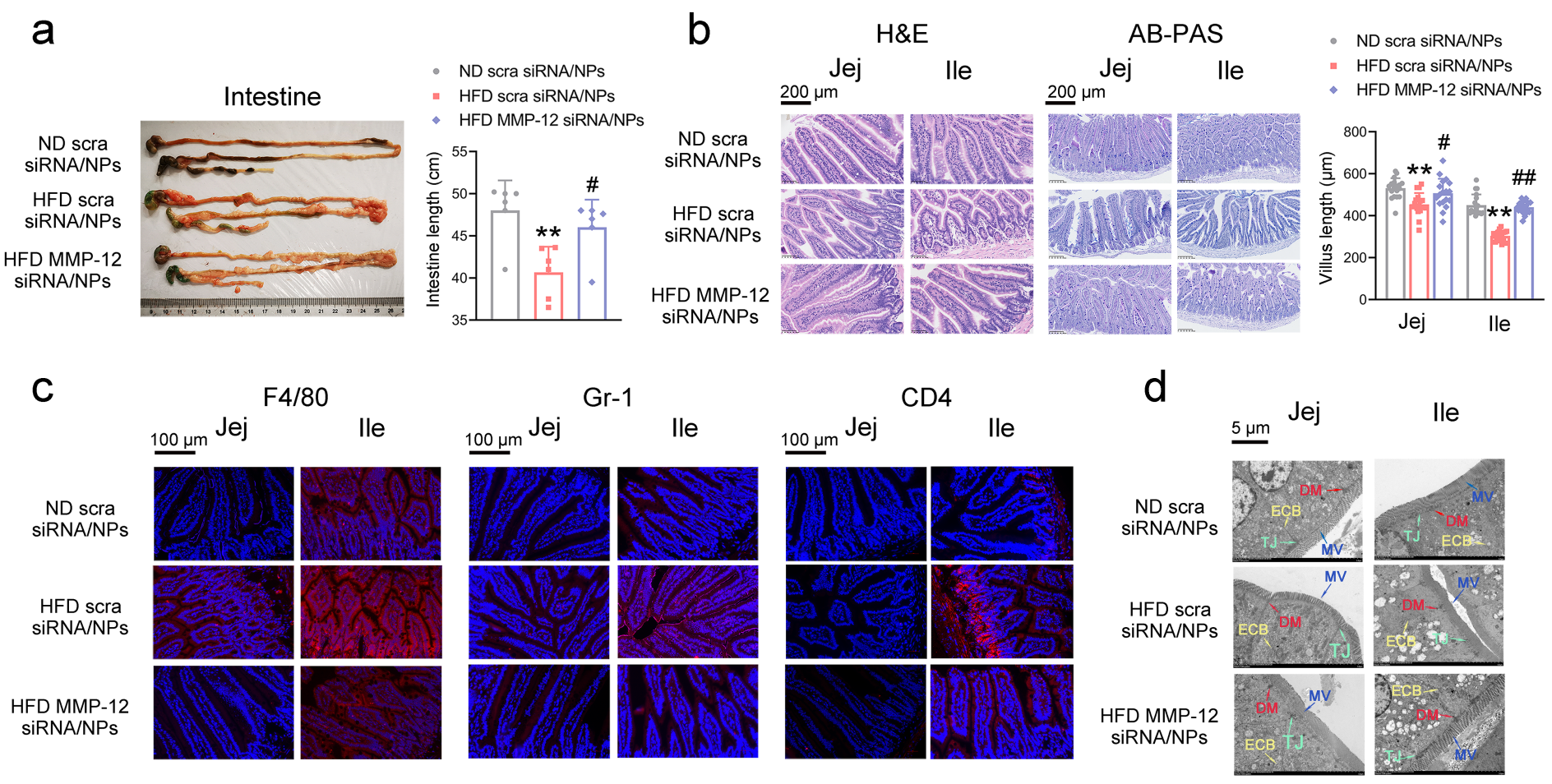

Ile
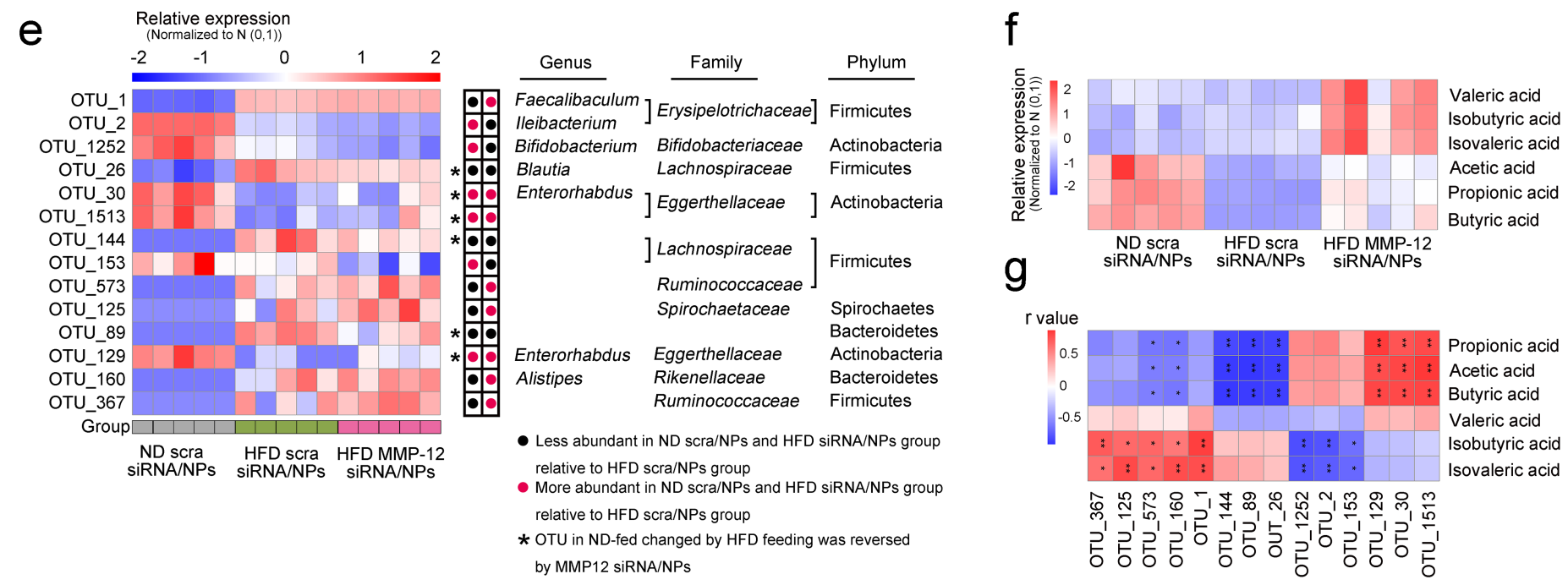

\section{Figure 7}

MMP-12-siRNA-CPA NPs attenuate inflammation and maintain the permeability of mouse small intestines. (a) Typical shape (left) and length (right) of the small intestines. (b) H\&E and AB-PAS staining of small intestines (scale bars: $200 \mu \mathrm{m}$ ). (c) F4/80, Gr-1 and CD4 expression in the small intestines was assessed by immunofluorescence staining (scale bars: $100 \mu \mathrm{m}$ ). (d) TEM analysis of jejunum (left) and ileum (right) epithelial cells. Key morphological features, such as the microvilli (MV), TJ, epithelial cell border (ECB), and desmosome (DM), are indicated (scale bars: $5 \mu \mathrm{m}$ ). For a-d, $\mathrm{n}=6$ for each group. ${ }^{*} p<0.05,{ }^{*} p<0.01$ vs. HFD scra siRNA/NP group, \#p<0.05, \#\#p<0.01 vs. HFD MMP-12 siRNA/NP group. All data are presented as the mean \pm SD. One-way ANOVA followed by Tukey's multiple comparisons test. 
(e) Abundance heatmap of 14 OTUs significantly changed by MMP-12 siRNA/NPs in HFD-fed mice based on RDA, $n=5$ for each group. (f) Abundance heatmap of significantly changed SCFAs, $n=5$ for each group.

(g) Heatmap of Spearman correlation analysis between changed OTUs and SCFAs. ${ }^{\star} p<0.05,{ }^{* \star} p<0.01$. Jej: jejunum; lle: ileum.

a

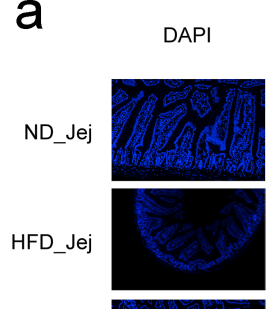

พ⿻上丨)

HFD_lle
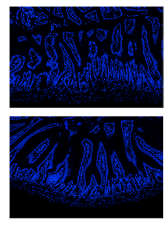

b
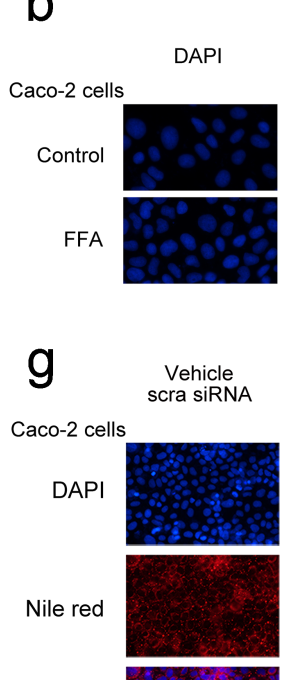

Merge
MMP-12
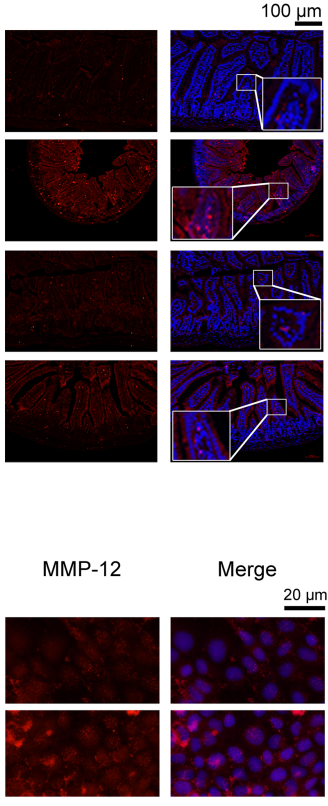

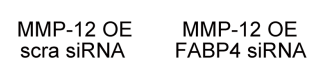
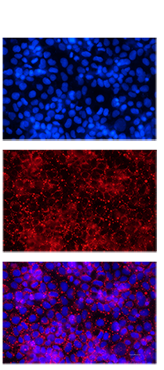

FFA treatment

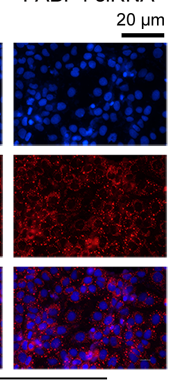

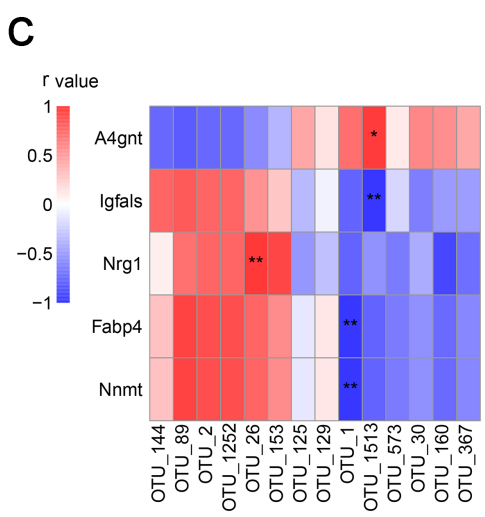

d

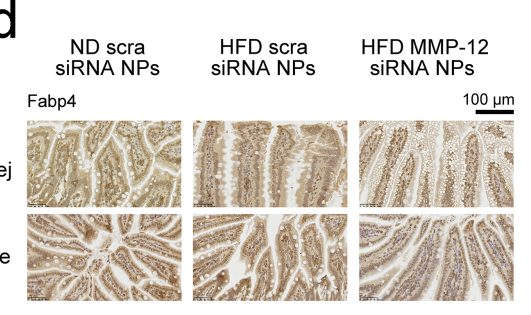

$\mathrm{h}$
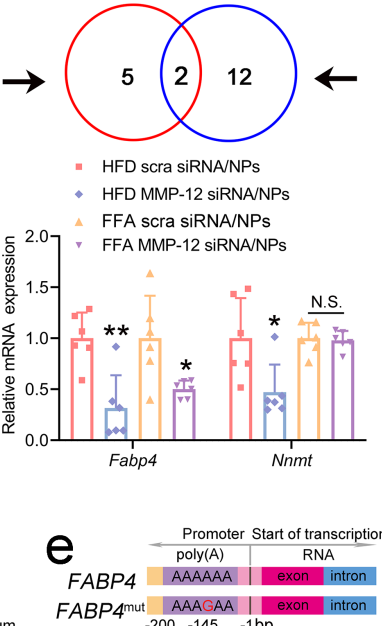

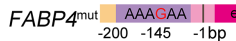

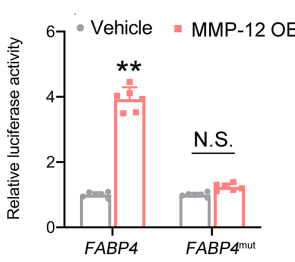

\section{i}

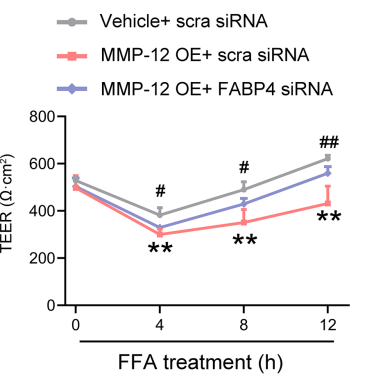

\section{Figure 8}

FABP4 serves as an effector of MMP-12 in the small intestines of HFD-fed mice. $(a, b)$ Immunofluorescence analysis of cytosolic and nuclear MMP-12 protein expression in mouse small intestines (jejunum and ileum) (scale bars: $100 \mu \mathrm{m}$ ) and Caco-2 cells (scale bars: $20 \mu \mathrm{m}$ ). (c) Heatmap of Spearman correlation analysis between changed small intestinal gene expression and OTUs (left), ${ }^{*} p<0.05,{ }^{* *} p<0.01$. Heatmap of Spearman correlation analysis between changed small intestinal gene expression and SCFA contents (right), ${ }^{*} p<0.05,{ }^{*} p p<0.01$. Venn diagrams and RT-qPCR validation of correlation analyses (middle), ${ }^{*} p<0.05,{ }^{*} p<0.01$ vs. HFD scra siRNA/NPs or FFA scra siRNA/NPs group. (d) IHC analysis of Fabp4 expression (scale bars: $100 \mu \mathrm{m}$ ). (e) Luciferase activity of the FABP4 promoter or FABP4mut promotor in Caco-2 cells transfected with vehicle or MMP-12 plasmids, $n=6$ for each group. (f) ChIP assays with the indicated antibodies in Caco-2 cells transfected with vehicle or MMP-12 plasmid, 
$\mathrm{n}=3$ for each group. ${ }^{\star *} \mathrm{p}<0.01$ vs. vehicle group. Caco-2 cells were transfected with the indicated plasmid or siRNA for $24 \mathrm{~h}$ and then treated with $0.4 \mathrm{mM}$ FFAs for another $24 \mathrm{~h}$. $(\mathrm{g})$ Nile red staining (scale bars: 20 $\mu \mathrm{m})$. (h) Supernatant IL-6, MCP-1 and TNF-a levels. (i) The TEER levels, $\mathrm{n}=5$ for each group. ${ }^{*} \mathrm{p}<0.05$, ${ }^{\star *} p<0.01$ vs. Vehicle + scra siRNA group, $\# p<0.05, \# \# p<0.01$ vs. MMP-12 overexpression (OE) + Fabp4 siRNA group. All data are presented as the mean \pm SD. One-way ANOVA followed by Tukey's multiple comparisons test. N.S.: no significance; Jej: jejunum; lle: ileum.

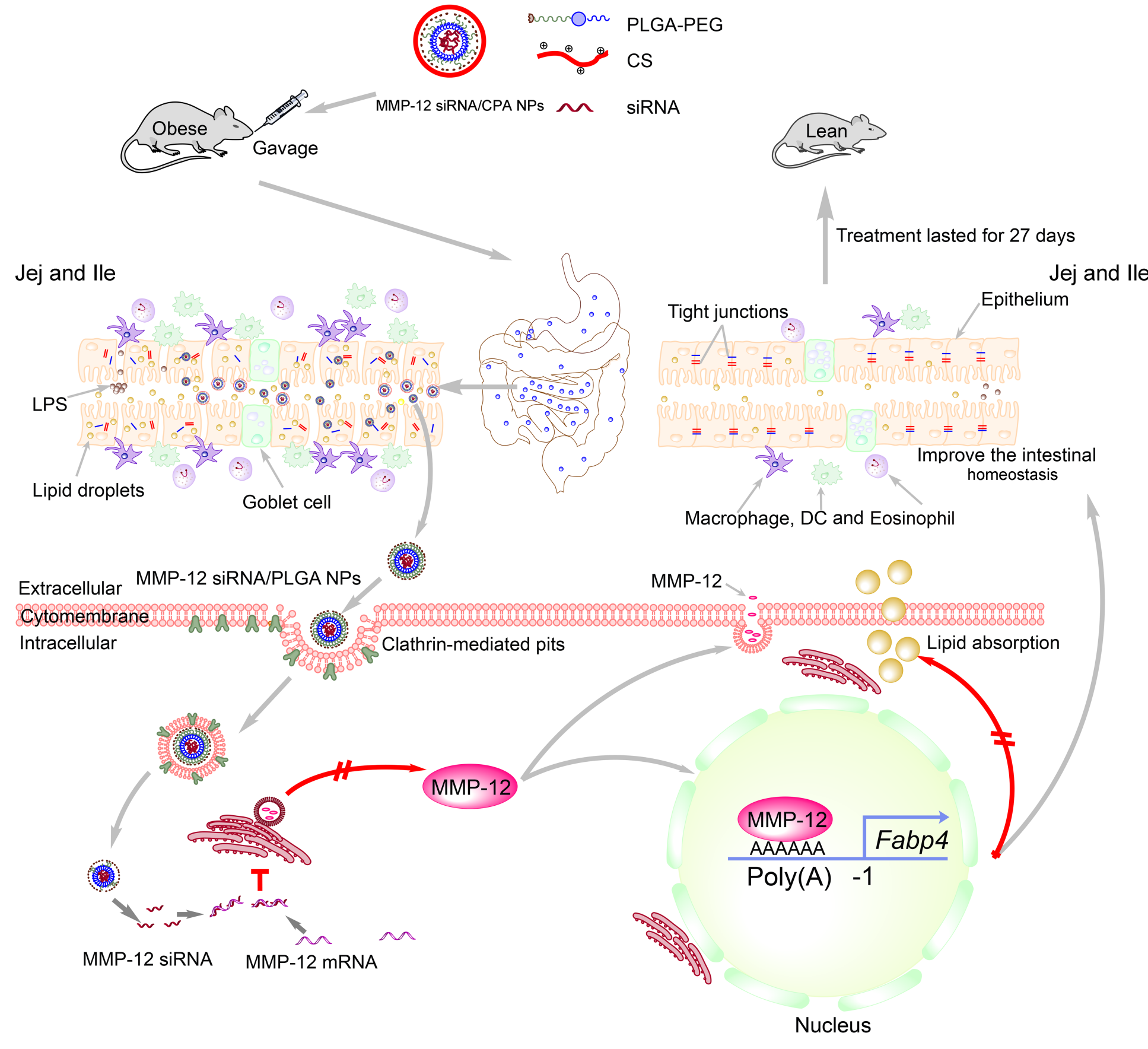

Figure 9

A schematic model illustrating the transcriptional regulation of microenvironment homeostasis by oral NP-mediated delivery of MMP-12 siRNA in mouse small intestines. 


\section{Supplementary Files}

This is a list of supplementary files associated with this preprint. Click to download.

- SupplementaryFile.docx 